University of Nebraska - Lincoln

DigitalCommons@University of Nebraska - Lincoln

Agronomy \& Horticulture - Faculty Publications

Agronomy and Horticulture Department

2009

\title{
Quantum Simulation of High-Order Harmonic Spectra of the Hydrogen Atom
}

\author{
A. D. Bandrauk \\ Département de Chimie, Faculté des Sciences, Université de Sherbrooke, Sherbrooke, Quebéc, Canada \\ S. Chelkowski \\ Département de Chimie, Faculté des Sciences, Université de Sherbrooke, Sherbrooke, Quebéc, Canada \\ Dennis J. Diestler \\ University of Nebraska-Lincoln, ddiestler1@unl.edu \\ J. Manz \\ Institut für Chemie und Biochemie, Freie Universität Berlin, 14195 Berlin, Germany \\ K.-J. Yuan \\ Département de Chimie, Faculté des Sciences, Université de Sherbrooke, Sherbrooke, Quebéc, Canada
}

Follow this and additional works at: https://digitalcommons.unl.edu/agronomyfacpub

Part of the Plant Sciences Commons

Bandrauk, A. D.; Chelkowski, S.; Diestler, Dennis J.; Manz, J.; and Yuan, K.-J., "Quantum Simulation of HighOrder Harmonic Spectra of the Hydrogen Atom" (2009). Agronomy \& Horticulture -- Faculty Publications. 352.

https://digitalcommons.unl.edu/agronomyfacpub/352

This Article is brought to you for free and open access by the Agronomy and Horticulture Department at DigitalCommons@University of Nebraska - Lincoln. It has been accepted for inclusion in Agronomy \& Horticulture -Faculty Publications by an authorized administrator of DigitalCommons@University of Nebraska - Lincoln. 


\title{
Quantum simulation of high-order harmonic spectra of the hydrogen atom
}

\author{
A. D. Bandrauk, ${ }^{1}$ S. Chelkowski, ${ }^{1}$ D. J. Diestler, ${ }^{2,3}$ J. Manz, ${ }^{3}$ and K.-J. Yuan ${ }^{1,3, *}$ \\ ${ }^{1}$ Département de Chimie, Faculté des Sciences, Université de Sherbrooke, Sherbrooke, Quebéc, Canada J1K $2 R 1$ \\ ${ }^{2}$ Department of Agronomy and Horticulture, University of Nebraska-Lincoln, Lincoln, Nebraska 68583, USA \\ ${ }^{3}$ Institut für Chemie und Biochemie, Freie Universität Berlin, 14195 Berlin, Germany
}

(Received 22 May 2008; published 3 February 2009)

\begin{abstract}
Three alternative forms of harmonic spectra, based on the dipole moment, dipole velocity, and dipole acceleration, are compared by a numerical solution of the Schrödinger equation for a hydrogen atom interacting with a linearly polarized laser pulse, whose electric field is given by $E(t)=E_{0} f(t) \cos \left(\omega_{0} t+\eta\right)$ with Gaussian carrier envelope $f(t)=\exp \left(-t^{2} / \delta^{2}\right)$. The carrier frequency $\omega_{0}$ is fixed to correspond to a wavelength of $800 \mathrm{~nm}$. Spectra for a selection of pulses, for which the intensity $I_{0}=c \varepsilon_{0} E_{0}^{2}$, duration $T \propto \delta$, and carrier-envelope phase $\eta$ are systematically varied, show that, depending on $\eta$, all three forms are in good agreement for "weak" pulses with $I_{0}<I_{b}$, the over-barrier ionization threshold, but that marked differences among the three appear as the pulse becomes shorter and stronger $\left(I_{0}>I_{b}\right)$. Except for scalings by powers of the harmonic frequency, the three forms differ from one another only by "limit contributions" proportional to the expectation values of the dipole moment $\left\langle z\left(t_{f}\right)\right\rangle$ or dipole velocity $\left\langle\dot{z}\left(t_{f}\right)\right\rangle$ at the end $\left(t_{f}\right)$ of the pulse. For long, weak pulses the limit contributions are negligible, whereas for short, strong ones they are not. In the short, strong limit, where $\left\langle\dot{z}\left(t_{f}\right)\right\rangle \neq 0$ and therefore $\langle z(t)\rangle$ may increase without bound (i.e., the atom may ionize), depending on $\eta$, an "infinite-time" spectrum based on the acceleration form provides a convenient computational pathway to the corresponding infinite-time dipole-velocity spectrum, which is related directly to the experimentally measured "harmonic photon number spectrum" (HPNS). For short, intense pulses the HPNS is quite sensitive to $\eta$ and exhibits not only the usual odd harmonics but also even ones. The analysis also reveals that most of the harmonic photons are emitted during the passage of the pulse. Because of the divergence of $\langle z(t)\rangle$ the dipolemoment form does not provide a numerically reliable route to the harmonic spectrum for very short (fewcycle), very intense laser pulses.
\end{abstract}

DOI: 10.1103/PhysRevA.79.023403

PACS number(s): 42.65.Ky, 32.80.Wr, 42.50.Hz

\section{INTRODUCTION}

High-harmonic generation (HHG) - the phenomenon in which low-frequency (infrared) radiation is converted, by the interaction of a laser pulse with a low-density gas, for example, to higher frequencies that are integer multiples (harmonics) of the low frequency-holds promise as a source of intense, coherent, short-wavelength radiation for such new practical applications as time-dependent $\mathrm{x}$-ray scattering [1] and attosecond pulse generation [2]. Most theoretical descriptions of HHG consider a single atom (or molecule), which is usually assumed to have a single "active" valence electron driven by an external electric field representing the laser pulse (see, however, Ref. [3]). The frequency distribution of the harmonic radiation, to which we shall henceforth loosely refer in the present context as the "harmonic spectrum," is variously described for single atoms as being proportional to the squared magnitude of the Fourier transform (FT) of the expectation value of either the dipole moment [4-12], the dipole acceleration [1,8,9,11-19], or the dipole velocity $[12,20]$. We are aware of just a single previous comparison of dipole, dipole-velocity, and dipole-acceleration forms based on accurate solutions of the time-dependent Schrödinger equation, but this was restricted to rates of harmonic generation by periodic, or continuous-wave fields at

\footnotetext{
*Corresponding author. Email address: kaijun.yuan@usherbrooke.ca
}

low intensity and infinite duration [21]. Expressions for the harmonic spectrum that are proportional to the (double) FT of the time-autocorrelation function of the dipole moment [4] or the dipole velocity [20] scale as the square of the density of the gas, reflecting the cooperativity of HHG, as observed, for example, by Lorin et al. [22], who employed coupled Maxwell-Schrödinger equations to simulate HHG in a one-dimensional model of $\mathrm{H}_{2}^{+}$gas.

An important work in the present context is that of Burnett et al. [13]. Alluding to a remark by Sundaram and Milonni [4] that the total power radiated by an atomic dipole is proportional to the expectation value of the squared acceleration, they questioned the use of the dipole form of the harmonic spectrum, implying that the correct form is proportional to the FT of the squared magnitude of the expectation value of the dipole acceleration. By means of integration by parts, they derived a relationship between the FTs of the dipole and dipole acceleration, which involve limit contributions depending on the expectation values of the dipole moment and dipole velocity at the end of the laser pulse. They compared "power spectra" for a one-dimensional model atom, finding good agreement between dipole and dipoleacceleration spectra at low intensity, but marked differences between them at high intensity. Stating that it is impractical to compute the dipole-acceleration spectrum by correcting the dipole-moment spectrum with the limit contributions, Burnett et al. recommended calculating the acceleration spectrum directly. Some researchers have subsequently followed their advice, while others have adhered to the dipole 
form, or in some cases have used both. For example, numerically accurate simulations of HHG for the three-dimensional (3D) hydrogen-molecular ion show that the acceleration form is preferable for the strong-field approximation to angular distributions of the harmonic radiation [9]. However, other simulations of HHG for a one-electron 3D diatomic molecule, based on the strong-field approximation [7], indicated that the dipole-velocity form of the strong-field limit "gives the closest agreement with exact results" [12].

The purpose of this paper is to explore the connections among the three forms (i.e., dipole-moment, dipole-velocity, and dipole-acceleration) of the harmonic spectrum that have been considered previously [1,3-19]. We present and compare the three forms computed from accurate numerical solutions of the Schrödinger equation for the interaction of a hydrogen atom $(\mathrm{H})$ with a linearly polarized laser pulse. Note that for the present purpose we invoke no approximations (e.g., the strong-field approximation [7,12]), because they may affect the results for the three forms differently. We fix the carrier frequency of the pulse. We vary the intensity from "low" $\left(I_{0}<I_{b}\right.$, the over-barrier ionization threshold) to "high" $\left(I_{0}>I_{b}\right)$. Motivated by recent experimental advances in the production of single-cycle pulses [23], we vary the duration from 16 down to 3 optical cycles. Likewise, results of a "double-slit" experiment in the attosecond time domain [24], which are quite sensitive to the carrier-envelope phase (CEP) of the laser pulse, have induced us to examine the influence of the CEP on the harmonic spectra. Special attention is paid to the dipole-velocity form, which is directly related to the "harmonic photon number spectrum" (HPNS) [20] measured experimentally for dilute atomic gases.

\section{THEORY}

Consider a linearly $(z-)$ polarized laser pulse with $z$ component of the electric field $E(t)$ interacting with an $\mathrm{H}$ atom. The Hamiltonian governing the motion of the electron relative to the nucleus can be expressed as

$$
H(t)=\frac{\mathbf{p}^{2}}{2}-\frac{1}{r}+z E(t)=H_{a}+W(t),
$$

where $H_{a} \equiv \mathbf{p}^{2} / 2-1 / r$ refers to the isolated atom. The form of the matter-radiation interaction indicates that the description is cast in the length gauge in the long-wavelength approximation. Here and below, except where it is stated otherwise, we employ atomic units: $a_{0}, \hbar / m_{e} a_{0}$, and $\hbar^{2} / m_{e}^{2} a_{0}^{3}$ for length, velocity, and acceleration, respectively; $E_{h} / e a_{0}$ for the electric field; and $\left(e a_{0}\right)^{2}$ for harmonic spectra. $\left(m_{e}\right.$ stands for the mass of the electron, $e$ for the magnitude of its charge, $a_{0}$ for the Bohr radius, and $\hbar$ for Planck's (modified) constant; $E_{h} \equiv \hbar^{2} / m_{e} a_{0}^{2}$.)

The wave packet describing the relative motion in spherical polar coordinates satisfies the Schrödinger equation

$$
\begin{aligned}
i \frac{\partial \Psi(r, \theta, \phi, t)}{\partial t}= & \left\{-\frac{1}{2 r^{2}} \frac{\partial}{\partial r}\left(r^{2} \frac{\partial}{\partial r}\right)+\frac{\mathbf{L}^{2}}{2 r^{2}}-\frac{1}{r}\right. \\
& +r \cos \theta E(t)\} \Psi(r, \theta, \phi, t),
\end{aligned}
$$

where $\mathbf{L}$ is the orbital angular momentum. We assume that the $\mathrm{H}$ atom occupies the ground state $(1 s)$ initially (i.e., at $\left.t=t_{i}\right)$. Since the potential energy to which the electron is subject is cylindrically symmetric, as is the initial state of the atom, the wave packet must remain cylindrically symmetric as it evolves. The $z$ component of $\mathbf{L}$ therefore vanishes; that is, the magnetic quantum number $m$ is restricted to zero. As a consequence, the wave function can be represented in the (restricted) basis of normalized eigenfunctions of $\mathbf{L}^{2}$ as

$$
\Psi(\mathbf{r}, t)=\Psi(r, \theta, \phi, t)=\sum_{l} R_{l}(r, t) Y_{l 0}(\theta, \phi),
$$

where

$$
Y_{l 0}(\theta, \phi)=\sqrt{(2 l+1) / 4 \pi} P_{l}(\cos \theta) .
$$

Substituting Eq. (2.3) into Eq. (2.2) and projecting both members of the resulting equation onto $Y_{l 0}$ (i.e., multiplying both sides by the operator $\int d \Omega Y_{10}^{*} \ldots$, where $d \Omega$ $=\sin \theta d \theta d \phi$ represents the element of solid angle), we obtain the following set of coupled second-order partial differential equations for the radial wave functions

$$
\begin{aligned}
i \frac{\partial R_{l}(r, t)}{\partial t}= & \left\{-\frac{1}{2 r^{2}} \frac{\partial}{\partial r}\left(r^{2} \frac{\partial}{\partial r}\right)+\frac{l(l+1)}{2 r^{2}}-\frac{1}{r}\right\} R_{l}(r, t) \\
& +\frac{l r E(t) R_{l-1}(r, t)}{\sqrt{(2 l-1)(2 l+1)}}+\frac{(l+1) r E(t) R_{l+1}(r, t)}{\sqrt{(2 l+1)(2 l+3)}}, \\
l=0,1,2 \ldots, &
\end{aligned}
$$

where we have used Eq. (A2) of Appendix A. The $\left\{R_{l}(r, t)\right\}$ are subject to the initial conditions

$$
R_{l}\left(r, t_{i}\right)=\left\{\begin{array}{cc}
2 e^{-r}, & l=0 \\
0, & l \geqslant 1,
\end{array}\right.
$$

where $2 e^{-r}$ is the normalized radial factor of the $1 s$ wave function.

We describe the harmonic spectrum $D(\omega)$ in three alternative forms: $D(\omega)$ is equal to the squared magnitude of the FT of the expectation value of either the dipole moment $(-z)$, the dipole velocity $(-\dot{z})$, or the dipole acceleration $(-\ddot{z})$. Thus, we set

$$
D_{\zeta}(\omega)=|\zeta(\omega)|^{2}
$$

where

$$
\zeta(\omega) \equiv \int_{t_{i}}^{t_{f}} d t \exp (-i \omega t)\langle\zeta(t)\rangle,
$$

and

$$
\langle\zeta(t)\rangle=\langle\Psi(t)|\zeta| \Psi(t)\rangle, \quad \zeta=-z,-\dot{z},-\ddot{z} .
$$

As indicated by Eq. (2.9), in atomic units the dipole moment $\zeta=-z$ is the negative of the $z$ coordinate of the electron relative to the nucleus. Since the harmonic spectra defined by Eqs. (2.7) and (2.8) do not depend on the sign of $\zeta$, for notational economy we suppress the minus sign, although we continue to refer to the coordinate $z$ loosely as the dipole moment, bearing in mind the absent minus sign. 
Using Eqs. (2.3), (2.4), and (2.9), we can write the expectation value of the dipole moment itself as

$$
\begin{aligned}
\langle z(t)\rangle & =\sum_{l} \sum_{k} \int_{0}^{\infty} d r r^{3} R_{l}^{*}(r, t) R_{k}(r, t) \int d \Omega Y_{l 0}^{*} \cos \theta Y_{k 0} \\
& =2 \sum_{l} \frac{(l+1)}{\sqrt{(2 l+1)(2 l+3)}} \operatorname{Re}\left[\int_{0}^{\infty} d r r^{3} R_{l}^{*}(r, t) R_{l+1}(r, t)\right],
\end{aligned}
$$

where the second line of Eq. (2.10) follows from the properties of the Legendre polynomials [see Appendix A, Eq. (A2)].

The dipole velocity is given by

$$
\dot{z}=(i)^{-1}[z, H]=\partial H / \partial p_{z}=p_{z},
$$

where $p_{z}$ is the $z$ component of the relative momentum and the last member of Eq. (2.11) depends on Eq. (2.1). Hence the expectation value of the dipole velocity can be written

$$
\langle\dot{z}(t)\rangle=\left\langle\Psi(t)\left|p_{z}\right| \Psi(t)\right\rangle=-i \int d \mathbf{r} \Psi^{*}(\mathbf{r}, t) \frac{\partial}{\partial z} \Psi(\mathbf{r}, t) .
$$

Using Eq. (2.3) and the relation

$$
\frac{\partial}{\partial z}=\cos \theta \frac{\partial}{\partial r}-\frac{\sin \theta}{r} \frac{\partial}{\partial \theta},
$$

we can rewrite Eq. (2.12) as

$$
\begin{aligned}
\langle\dot{z}(t)\rangle= & -i \sum_{l} \sum_{k} \int_{0}^{\infty} d r r^{2} \int d \Omega R_{l}^{*}(r, t) Y_{l 0}^{*} \\
& \times\left[\cos \theta \frac{\partial}{\partial r}-\frac{\sin \theta}{r} \frac{\partial}{\partial \theta}\right] R_{k}(r, t) Y_{k 0} .
\end{aligned}
$$

A lengthy analysis (see Appendix B) yields

$$
\begin{aligned}
\langle\dot{z}(t)\rangle= & 2 \sum_{l} \frac{(l+1)}{\sqrt{(2 l+1)(2 l+3)}} \\
& \times\left\{\operatorname{Im}\left[\int_{0}^{\infty} d r r^{2} \frac{\partial R_{l}(r, t)}{d r} R_{l+1}^{*}(r, t)\right]\right. \\
& \left.-l \operatorname{Im}\left[\int_{0}^{\infty} d r r R_{l} R_{l+1}^{*}\right]\right\} .
\end{aligned}
$$

In analogy with Eq. (2.11), we can express the dipole acceleration as

$$
\ddot{z}=(i)^{-1}[\dot{z}, H]=(i)^{-1}\left[p_{z}, H\right]=-\partial H / \partial z .
$$

From the last member of Eq. (2.16) and Eq. (2.1) we deduce

$$
\ddot{z}=\frac{\partial r^{-1}}{\partial z}-E(t)=-z r^{-3}-E(t) .
$$

Combining Eqs. (2.3) and (2.17), we get

$$
\begin{aligned}
\langle\ddot{z}(t)\rangle= & -\left\langle\Psi(t)\left|z r^{-3}\right| \Psi(t)\right\rangle-E(t) \\
= & -\sum_{l} \sum_{k} \int_{0}^{\infty} d r R_{l}^{*}(r, t) R_{k}(r, t) \\
& \times \int_{l} d \Omega Y_{l 0}^{*} \cos \theta Y_{k 0}-E(t) \\
= & -2 \sum_{l} \frac{(l+1)}{\sqrt{(2 l+1)(2 l+3)}} \\
& \times \operatorname{Re}\left[\int_{0}^{\infty} d r R_{l}^{*}(r, t) R_{l+1}(r, t)\right]-E(t),
\end{aligned}
$$

where we utilize Eq. (A2) to obtain the third line of Eq. (2.18).

The three forms of harmonic spectra we are considering can be related to one another as follows. Using the definitions in Eqs. (2.8) and (2.9), we write the FT of $\dot{z}$ explicitly as

$$
\dot{z}(\omega)=\int_{t_{i}}^{t_{f}} d t e^{-i \omega t}\langle\dot{z}(t)\rangle .
$$

Integrating by parts, we obtain

$$
\dot{z}(\omega)=e^{i \omega t_{f}}\left\langle z\left(t_{f}\right)\right\rangle+i \omega z(\omega),
$$

where the condition $\left\langle z\left(t_{i}\right)\right\rangle=0$, which follows from the spherical symmetry of the initial state, is implicit. From Eqs. (2.7) and (2.20) we deduce the relationship between the dipole and dipole-velocity forms of the harmonic spectrum

$$
D_{\dot{z}}(\omega)=\left\langle z\left(t_{f}\right)\right\rangle^{2}-2 \omega\left\langle z\left(t_{f}\right)\right\rangle \operatorname{Im}\left[e^{i \omega t_{f}} z(\omega)\right]+\omega^{2} D_{z}(\omega),
$$

where the limit contribution of the dipole moment $\left\langle z\left(t_{f}\right)\right\rangle$ is manifest. The alternative integration by parts of $\dot{z}(\omega)$ yields

$$
\ddot{z}(\omega)=i \omega \dot{z}(\omega)-e^{i \omega t_{f}}\left\langle\dot{z}\left(t_{f}\right)\right\rangle,
$$

where we have invoked the symmetry-dictated condition $\left\langle\dot{z}\left(t_{i}\right)\right\rangle=0$. [Note that this condition differs from that imposed in the tunneling-ionization model [25], which assumes the instantaneous initial condition $\dot{z}\left(t_{i}\right)=0$; see the discussion just above Eq. (4.1).] Using Eqs (2.7) and (2.22), we obtain the following relationship between the dipole-velocity and dipole-acceleration forms of the harmonic spectrum:

$$
D_{\ddot{z}}(\omega)=-\left\langle\dot{z}\left(t_{f}\right)\right\rangle^{2}+2\left\langle\dot{z}\left(t_{f}\right)\right\rangle \operatorname{Re}\left[e^{i \omega t_{f}} \ddot{z}(\omega)\right]+\omega^{2} D_{\dot{z}}(\omega) .
$$

In Eq. (2.23) the limit contribution of the dipole velocity appears, in analogy with that of the dipole itself in Eq. (2.21).

From Eqs. (2.21) and (2.23) it is clear that the three forms of harmonic spectra (with proper scalings by powers of $\omega$ ) may be identical, or nearly so, or may differ radically, depending on the values of $\left\langle z\left(t_{f}\right)\right\rangle$ and $\left\langle\dot{z}\left(t_{f}\right)\right\rangle$ (i.e., whether these values are zero, or nearly so, or are quite different from zero). We show that $\left\langle z\left(t_{f}\right)\right\rangle$ and $\left\langle\dot{z}\left(t_{f}\right)\right\rangle$ depend significantly on the parameters that specify the laser pulse (e.g., the duration, intensity, and CEP). Suppose, for example, that $\left\langle z\left(t_{f}\right)\right\rangle$ 
$\approx\left\langle\dot{z}\left(t_{f}\right)\right\rangle \approx 0$. Then the three forms are simply related by the expression

$$
D_{\ddot{z}}(\omega) \approx \omega^{2} D_{\dot{z}}(\omega) \approx \omega^{4} D_{z}(\omega) .
$$

The expression $D_{\ddot{z}}(\omega) \approx \omega^{4} D_{z}(\omega)$ is implicit in relations given by Burnett et al. [13]. We note that all members of Eq. (2.24) have dimensions $Q^{2} L^{2} T^{-2}$ (where $Q$ stands for charge, $L$ for length, and $T$ for time). Following a previously established convention [19], we define the various harmonic spectra by

$$
\begin{gathered}
P_{z}(\omega) \equiv D_{z}(\omega) / T^{2}, \\
P_{\dot{z}}(\omega) \equiv D_{\dot{z}}(\omega) / T^{2} \omega^{2}, \\
P_{\ddot{z}}(\omega) \equiv D_{\ddot{z}}(\omega) / T^{2} \omega^{4},
\end{gathered}
$$

where $T \equiv t_{f}-t_{i}$ is the pulse duration. All of the harmonic spectra now have dimensions $Q^{2} L^{2}$. Note that $P_{\dot{z}}(\omega)$ corresponds to the HPNS [20], whereas $P_{z}(\omega)$ and $P_{z z}(\omega)$ refer to complementary, albeit related, dynamic properties based on $\langle z(t)\rangle$ and $\langle\ddot{z}(t)\rangle$, respectively [26].

We take the electric field of the laser pulse to be

$$
E(t)=\left\{\begin{array}{cc}
E_{0} f(t) \cos \left(\omega_{0} t+\eta\right), & t_{i}<t<t_{f} \\
0, & \text { elsewhere }
\end{array}\right.
$$

where the carrier envelope is of Gaussian form

$$
f(t)=\exp \left(-t^{2} / \delta^{2}\right) .
$$

Note that $f$ is symmetric about $t=0$. We set $t_{f} \simeq-t_{i}$ and choose the CEP $\eta$ so that the condition

$$
\int_{t_{i}}^{t_{f}} d t E(t)=0
$$

holds [27]. The duration of one optical cycle is $\tau=2 \pi / \omega_{0}$; the wavelength is $\lambda=\tau c$, where $c$ is the speed of light.

We note that the maximum electric field given by Eq. (2.26) and the corresponding maximum intensity $\left(I=c \varepsilon_{0} E^{2}\right.$, where $\varepsilon_{0}$ is the electric permittivity of vacuum) [28] depend on $\eta$. (Here and for the remainder of this Section we use SI units.) Likewise, the ponderomotive energy

$$
U_{p}=e^{2} E^{2} / 4 m_{e} \omega_{0}^{2},
$$

and hence the so-called Keldysh limit for ionization [29]

$$
\gamma=\sqrt{I_{p} / 2 U_{p}},
$$

where $I_{p}$ is the ionization potential $\left(=0.5 E_{\mathrm{h}}\right.$ for the ground state of the $\mathrm{H}$ atom), as well as the cutoff number [25]

$$
N_{m}=\left(I_{p}+3.17 U_{p}\right) / \hbar \omega_{0}
$$

[i.e., the number of the harmonic beyond which $P_{\ddot{z}}(\omega)$ rapidly falls off] depend on $\eta$. For reference, we characterize the intensity of a given pulse in terms of the maximum electric field $\left(E_{0}\right)$ by

$$
I_{0}=c \varepsilon_{0} E_{0}^{2}
$$

for $\eta=0$. The full width at half maximum of the carrier envelope of the intensity (i.e., $[f(t)]^{2}$ ) is

$$
\mathrm{FWHM}=\delta(2 \ln 2)^{1 / 2} .
$$

\section{NUMERICAL METHODS}

For convenience we rewrite Eq. (2.5) in terms of auxiliary radial wave functions defined by

$$
R_{l}(r, t) \equiv r^{-1} \psi_{l}(r, t) .
$$

Substitution of Eq. (3.1) into Eq. (2.5) yields

$$
\begin{aligned}
& i \frac{\partial \psi_{l}(r, t)}{\partial t}=\left\{-\frac{1}{2} \frac{\partial^{2}}{\partial r^{2}}+\frac{l(l+1)}{2 r^{2}}-\frac{1}{r}\right\} \psi_{l}(r, t) \\
&+\frac{\operatorname{lr} E(t) \psi_{l-1}(r, t)}{\sqrt{(2 l-1)(2 l+1)}}+\frac{(l+1) r E(t) \psi_{l+1}(r, t)}{\sqrt{(2 l+1)(2 l+3)}}, \\
& l=0,1,2 \ldots
\end{aligned}
$$

Equation (3.2) can be recast compactly in matrix form as

$$
i \frac{\partial \boldsymbol{\psi}(r, t)}{\partial t}=\mathbf{H}(r, t) \boldsymbol{\psi}(r, t)=\left[\mathbf{H}_{a}(r)+\mathbf{W}(r, t)\right] \boldsymbol{\psi}(r, t) .
$$

Here $\boldsymbol{\psi}$ is the column vector whose $l$ th element is $\psi_{l}(r, t) ; \mathbf{H}_{a}$ is the diagonal matrix with elements

$$
\left[\mathbf{H}_{a}\right]_{l l}=-\frac{1}{2} \frac{\partial^{2}}{\partial r^{2}}+\frac{l(l+1)}{2 r^{2}}-\frac{1}{r} .
$$

The elements of the tridiagonal matrix $\mathbf{W}$, which represents the interaction of the electric field with the dipole, are given by

$$
[\mathbf{W}(r, t)]_{k l}= \begin{cases}\operatorname{lr} E(t) / \sqrt{(2 l-1)(2 l+1)}, & k=l-1 \\ (l+1) r E(t) / \sqrt{(2 l+1)(2 l+3)}, & k=l+1 \\ 0 & \text { otherwise. }\end{cases}
$$

The solution of Eq. (3.3) can be written formally as

$$
\boldsymbol{\psi}(r, t+\Delta t)=\mathbf{U}(t+\Delta t, t) \boldsymbol{\psi}(r, t) .
$$

The time-evolution operator $\mathbf{U}$ is given by

$$
\mathbf{U}(t+\Delta t, t)=P\left\{\exp \left(-i \int_{t}^{t+\Delta t} d t^{\prime}\left[\mathbf{H}_{a}+\mathbf{W}\left(t^{\prime}\right)\right]\right)\right\},
$$

where $P$ signifies the Dyson chronological operator [30]. To second order in $\Delta t, \mathbf{U}$ can be approximated by [31]

$$
\mathbf{U}(t+\Delta t, t) \cong e^{-i \mathbf{H}_{a} \Delta t / 2} e^{-i \mathbf{W}(t) \Delta t} e^{-i \mathbf{H}_{a} \Delta t / 2} .
$$

The vector $\psi(r, t)$ is propagated one step in time from $t$ to $t+\Delta t$ in three stages corresponding to the successive action of the three exponential operators in Eq. (3.8) [32,33]. In the first stage $\psi(r, t)$ evolves to $\psi^{(1)}(r)$ according to the relation 
$\boldsymbol{\psi}^{(1)}(r)=e^{-i \mathbf{H}_{a} \Delta t / 2} \boldsymbol{\psi}(r, t)$, which can be recast (approximately) in the Crank-Nicholson form [34]

$$
\left(1+i \mathbf{H}_{a} \Delta t / 4\right) \boldsymbol{\psi}^{(1)}(r)=\left(1-i \mathbf{H}_{a} \Delta t / 4\right) \boldsymbol{\psi}(r, t) .
$$

Note that since $\mathbf{H}_{a}$ is diagonal, Eqs. (3.9) decouple. We then solve each implicit differential equation for $\psi_{l}^{(1)}(r)$ by the finite-difference method [35]. The second derivative in $\mathbf{H}_{a}$ is approximated by the three-point central-difference formula. The $\left\{\psi_{l}^{(1)}(r)\right\}$ are required to vanish at $r=0$ and $r=r_{\max }$.

In the second stage $\psi^{(1)}(r)$ evolves to $\psi^{(2)}(r)$ through the electric-field-dipole interaction according to

$$
\boldsymbol{\psi}^{(2)}(r)=e^{-i \mathbf{W}(r, t) \Delta t} \boldsymbol{\psi}^{(1)}(r) .
$$

We diagonalize $\mathbf{W}(r, t)$ by the unitary transformation [36]

$$
\mathbf{S}^{\dagger}(r, t) \mathbf{W}(r, t) \mathbf{S}(r, t)=\mathbf{w}_{\text {diag }}(r, t),
$$

and substitute the "inverse" of Eq. (3.11), W( $r, t)$ $=\mathbf{S}(r, t) \mathbf{w}_{\text {diag }}(r, t) \mathbf{S}^{\dagger}(r, t)$, into Eq. (3.10) to get

$$
\boldsymbol{\psi}^{(2)}(r)=\mathbf{S}(r, t) e^{-i \mathbf{w}_{\mathrm{diag}}(r, t) \Delta t} \mathbf{S}^{\dagger}(r, t) \boldsymbol{\psi}^{(1)}(r) .
$$

Thus, we obtain $\boldsymbol{\psi}^{(2)}(r)$ by multiplying $\boldsymbol{\psi}^{(1)}(r)$ successively by the matrices $\mathbf{S}^{\dagger}, e^{-i \mathbf{w}_{\text {diag }}(r, t) \Delta t}$, and $\mathbf{S}$. In the third and final stage we propagate $\psi^{(2)}(r)$ to $\psi(r, t+\Delta t)$ by a second application of the Crank-Nicholson finite-difference technique. The radial finite-difference grid contains $n_{r}$ points, separated by distance $\Delta r$, so that $r_{\max }=n_{r} \Delta r ; \Delta r$ is fixed at $0.125 a_{0}$. The choice of $n_{r}$ is dictated essentially by the parameters of the laser pulse. The time step is fixed at $\Delta t=0.025 \hbar / E_{h}$ $=0.025 \times 24.2$ attoseconds.

To avoid spurious effects due to the reflection of the wave packet from the boundary at $r=r_{\max }$, we multiply $\Psi(\mathbf{r}, t)$ by a "mask function" [33]

$$
g(r)= \begin{cases}1, & r<r_{0} \\ \left\{\cos \left[\pi\left(r-r_{0}\right) / 2\left(r_{\max }-r_{0}\right)\right]\right\}^{1 / 8}, & r_{0}<r<r_{\max } .\end{cases}
$$

For all results reported here we set $r_{\max }-r_{0}=32 a_{0}$. The consequence of the wave packet's entering the "absorber" domain $\left[r_{0}, r_{\text {max }}\right]$ is that the probability of presence of the system in the complementary domain $\left[0, r_{0}\right]$, which is given by

$$
P_{r_{0}}(t)=\int_{0}^{r_{0}} d r r^{2} \int d \Omega|\Psi(r, \theta, \phi, t)|^{2}=\sum_{l} \int_{0}^{r_{0}} d r r^{2}\left|R_{l}(r, t)\right|^{2},
$$

falls below unity. We set the maximum value of $l$ to 80 and monitor $P_{r_{0}}(t)$ to assure that it remains equal to one.

Once the wave packet has been propagated to time $t$, the expectation values $\langle z(t)\rangle,\langle\dot{z}(t)\rangle$, and $\langle\ddot{z}(t)\rangle$ are computed by Eqs. (2.10), (2.15), and (2.18). [We note that for $\langle z(t)\rangle$ and $\langle\ddot{z}(t)\rangle$ the angular integrals are evaluated by means of $81-$ point Gauss-Legendre quadrature [37], whereas $\langle\dot{z}(t)\rangle$ is calculated directly from the expression given in Eq. (2.15).] The radial integrations, as well as the time integrations in the FTs [Eqs. (2.8)], are done by the trapezoidal rule.

As additional checks on the reliability of the numerical methods, we have verified that $\langle\dot{z}(t)\rangle$ and $\langle\ddot{z}(t)\rangle$ computed using the expressions given in Eqs. (2.15) and (2.18) agree with the corresponding numerical first and second derivatives of $\langle z(t)\rangle$. We have also verified that the numerical solutions satisfy the relations in Eqs. (2.21) and (2.23).

\section{RESULTS}

We have computed harmonic spectra for a selection of laser pulses at fixed wavelength $\lambda=800 \mathrm{~nm}$ (optical cycle time $\tau=2.67 \mathrm{fs}$ ). We examine the influence of variations of
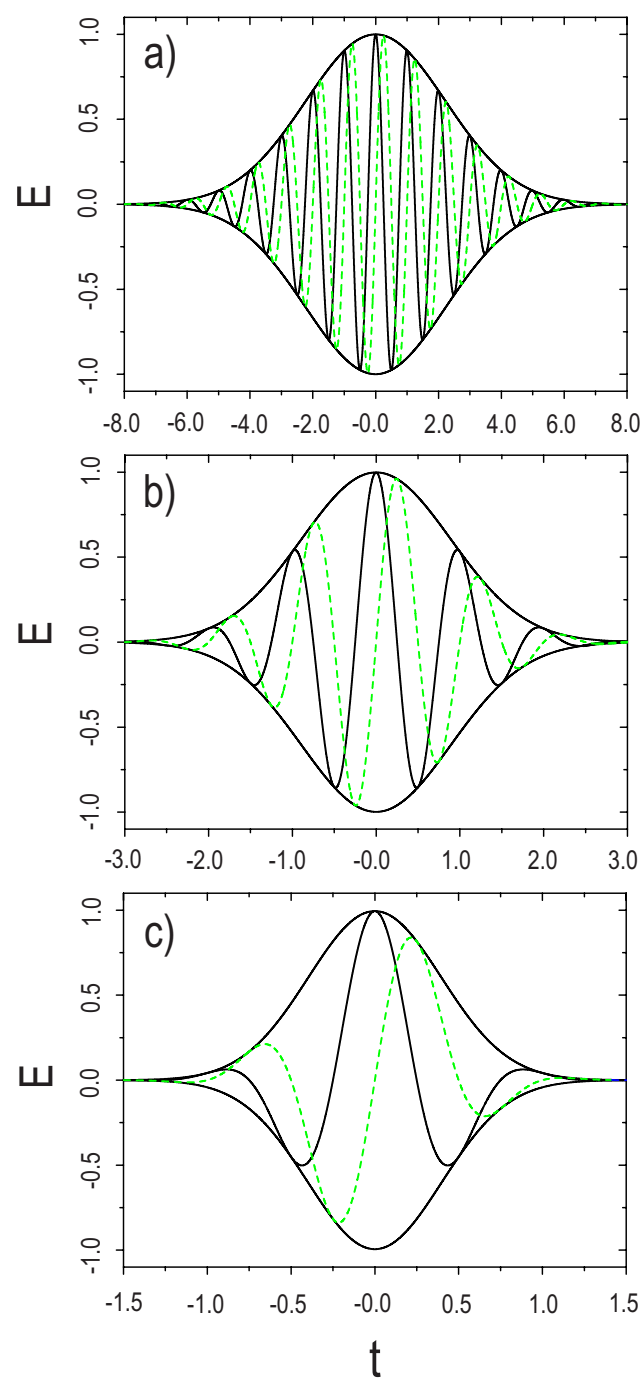

FIG. 1. (Color online) Electric field [in units of maximum amplitude $E_{0}$, defined in terms of the intensity by Eq. (2.32)] versus time $t$ (in units of optical-cycle time $\tau$ ) for selection of laser pulses specified by Eqs. (2.26)-(2.33). All pulses have wavelength $\lambda$ $=800 \mathrm{~nm}$, corresponding to optical-cycle time $\tau=2.67 \mathrm{fs}$. Durations of pulses shown in panels (a)-(c) are, respectively, $T=16 \tau, 6 \tau$, and $3 \tau$, corresponding to $\mathrm{FWHM}=9.33,3.99$, and $2.05 \mathrm{fs}$, respectively. Continuous and dashed lines correspond to CEPs $\eta=0$ and $-\pi / 2$, respectively. Electric-field amplitudes $E_{0}=0.0377 E_{h} / a_{0} e$ for weak (see Figs. 2-4) and $E_{0}=0.119 E_{h} / a_{0} e$ for strong (see Figs. 5-8) pulses correspond, respectively, to maximum intensities $I_{0}$ $=10^{14} \mathrm{~W} \mathrm{~cm}^{-2}\left(<I_{b}=1.4 \times 10^{14} \mathrm{~W} \mathrm{~cm}^{-2}\right.$, the threshold for overbarrier ionization) and $I_{0}=10^{15} \mathrm{~W} \mathrm{~cm}^{-2}\left(>I_{b}\right)$. Respective Keldysh parameters for weak and strong pulses are $\gamma=1.5$ and $\gamma=0.48$. 

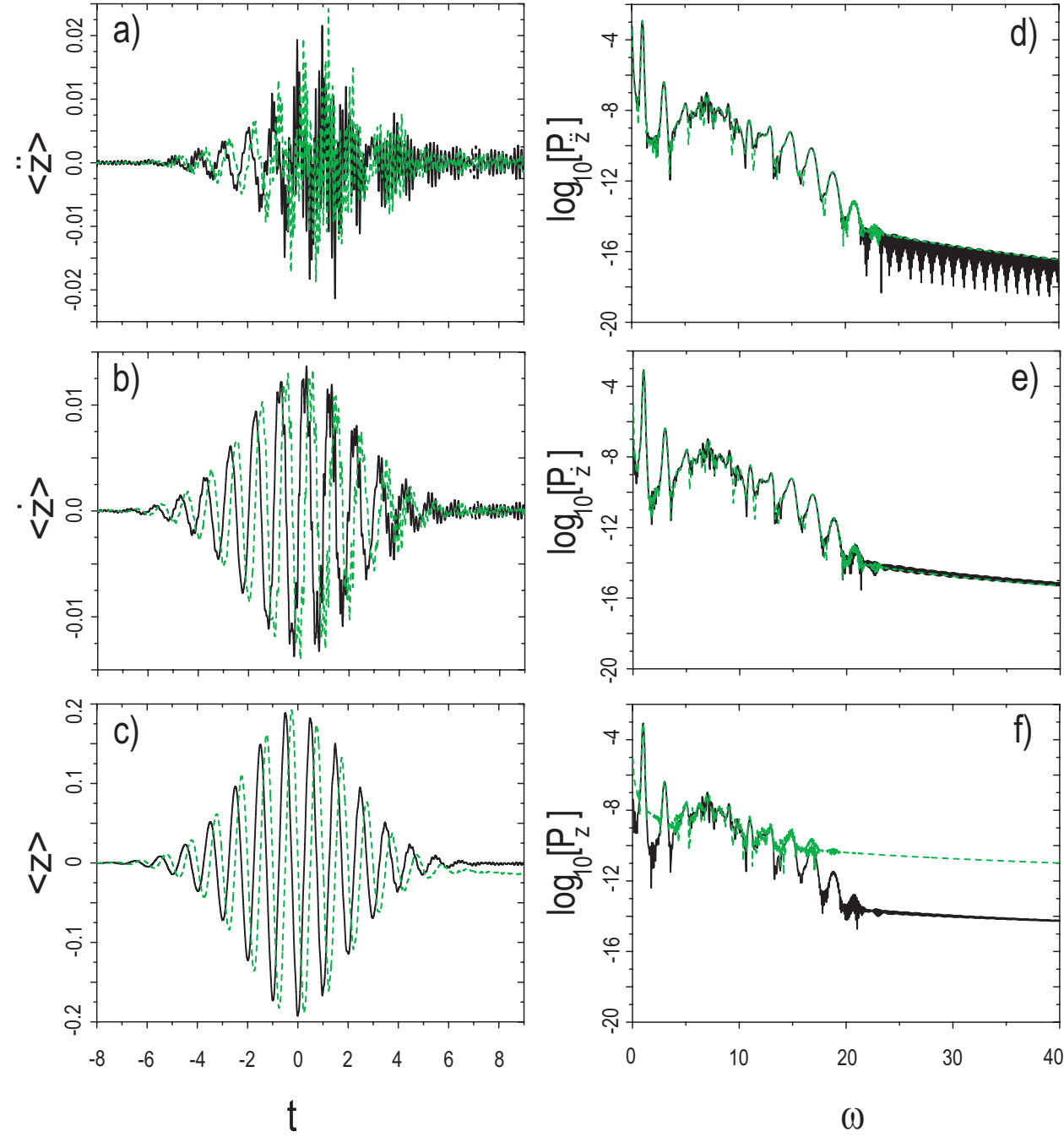

FIG. 2. (Color online) Expectation values (in atomic units) of dipole-acceleration $\langle\ddot{z}(t)\rangle \quad\left(e \hbar^{2} /\right.$ $\left.a_{0}^{3} m_{e}^{2}\right) \quad(\mathrm{a})$, dipole-velocity $\langle\dot{z}(t)\rangle$ $\left(e \hbar / a_{0} m_{e}\right)(\mathrm{b})$, and dipole moment $\langle z(t)\rangle\left(e a_{0}\right)$ (c) versus time $t$ (in units of optical cycles $\tau$ ), along with respective harmonic spectra $P_{\ddot{z}}(\omega)(\mathrm{d}), P_{\dot{z}}(\omega)(\mathrm{e})$, and $P_{z}(\omega)(\mathrm{f})$ [in atomic units of $\left(e a_{0}\right)^{2}$ as a function of frequency (in units of carrier frequency $\omega_{0}$ ), for longest weak pulse $\left(I_{0}=10^{14} \mathrm{~W} \mathrm{~cm}^{-2}, E_{0}\right.$ $\left.=0.0377 E_{h} / a_{0} e\right)$. Solid and dashed lines refer to CEPs $\eta=0$ and $\eta=-\pi / 2$, respectively [see Fig. 1(a)]. Initial time $t_{i}$ of pulse is set to $-8 \tau$, final time $t_{f}$ is chosen close to, but slightly larger than $-t_{i}$ such that $\left\langle\dot{z}\left(t_{f}\right)\right\rangle=0$. Actual duration of pulse is therefore slightly longer than $T=16 \tau$. intensity $I_{0}$, duration $T=t_{f}-t_{i}$, and CEP $\eta$. Plots of the electric field versus $t$ for the considered pulses are displayed in Fig. 1. The results, namely $\langle z(t)\rangle,\langle\dot{z}(t)\rangle$, and $\langle\ddot{z}(t)\rangle$ and the corresponding harmonic spectra $P_{z}(\omega), P_{\dot{z}}(\omega)$, and $P_{\ddot{z}}(\omega)$ are presented in Figs. 2-7. We consider two sets of results corresponding to (1) "weak" pulses $\left(I_{0}=10^{14} \mathrm{~W} \mathrm{~cm}^{-2}\right.$, below the over-barrier ionization threshold $I_{b}=1.4 \times 10^{14} \mathrm{~W} \mathrm{~cm}^{-2}$ ) with durations that correspond to 16,6 and 3 optical cycles $\tau$; (2) "strong" pulses $\left(I_{0}=10^{15} \mathrm{~W} \mathrm{~cm}^{-2}>I_{b}\right)$ of the same durations. For each of these six pulses we look at two CEPs, $\eta$ $=0$ and $\eta=-\pi / 2$.

Figure 2 shows the results for the longest weak pulses $(T=16 \tau, \mathrm{FWHM}=9.33 \mathrm{fs}, \eta=0,-\pi / 2)$. These pulses cause transient oscillations of rather low amplitude in $\langle z(t)\rangle,\langle\dot{z}(t)\rangle$, and $\langle\ddot{z}(t)\rangle$, as indicated in panels 2(c), 2(b), and 2(a), respectively. We note that the maxima in $\langle z(t)\rangle$ occur at the minima of the electric field, in accordance with adiabatic following at low intensity. After the passage of the pulse, $\langle z(t)\rangle$ remains oscillating with small amplitude about nearly constant values depending on $\eta$, which are close to, but not necessarily identical to, the initial value $\left\langle z\left(t_{i}\right)\right\rangle=0$. Consequently, both $\langle\dot{z}(t)\rangle$ and $\langle\ddot{z}(t)\rangle$ oscillate about zero with small amplitude. This allows us to define the final time $t_{f}$ as the time after $-t_{i}$ when $\left\langle\dot{z}\left(t_{f}\right)\right\rangle$ first vanishes (i.e., the pulse duration $T=t_{f}-t_{i}$ is taken to be just slightly larger than $-2 t_{i}$, which is $16 \tau$ for the present case).

The resulting harmonic spectra for the three forms are nearly identical, except for some significant deviations of $P_{z}(\omega)$ for $\eta=-\pi / 2$. Moreover, they are nearly insensitive to $\eta$, again with the exception of $P_{z}(\omega)$. The near identity of $P_{\ddot{z}}(\omega)$ and $P_{\dot{z}}(\omega)$ may be understood as a consequence of the vanishing of the dipole-velocity limit contribution (i.e., $\left.\left\langle\dot{z}\left(t_{f}\right)\right\rangle=0\right)$ in Eq. (2.23). Likewise, relation (2.21) suggests that $P_{\dot{z}}(\omega)$ and $P_{z}(\omega)$ would be identical if $\left\langle z\left(t_{f}\right)\right\rangle$ were equal to zero. Figure $2(\mathrm{c})$ shows that this condition is satisfied for $\eta=0$, in contrast with small negative deviations of $\left\langle z\left(t_{f}\right)\right\rangle$ from zero for $\eta=-\pi / 2$. As a consequence, $P_{z}(\omega)$ for $\eta$ $=-\pi / 2$ no longer exhibits the pronounced "falloff" [i.e., the precipitous drop in the magnitude of the spectrum for harmonics beyond $N_{m}$ defined by Eq. (2.31)] beginning at $N_{m}$ $=15$ that is evident in the other two spectra, regardless of $\eta$.

The results for the next shorter weak pulse $(T=6 \tau$, $\mathrm{FWHM}=3.99 \mathrm{fs}$ ) are illustrated in Fig. 3. The main findings are similar to those for the weak $T=16 \tau$ pulse, except for a significant influence of $\eta$, which is due to the fact that as $\eta$ departs from zero, the maximum in the electric field [see Fig. 1(b)] declines, causing $U_{p}$ and hence $N_{m}$ to decrease. The influence of $\eta$ becomes even more apparent for the shortest, weak pulse $[T=3 \tau, \mathrm{FWHM}=2.05 \mathrm{fs}$, see Fig. 1(c)]. Equa- 

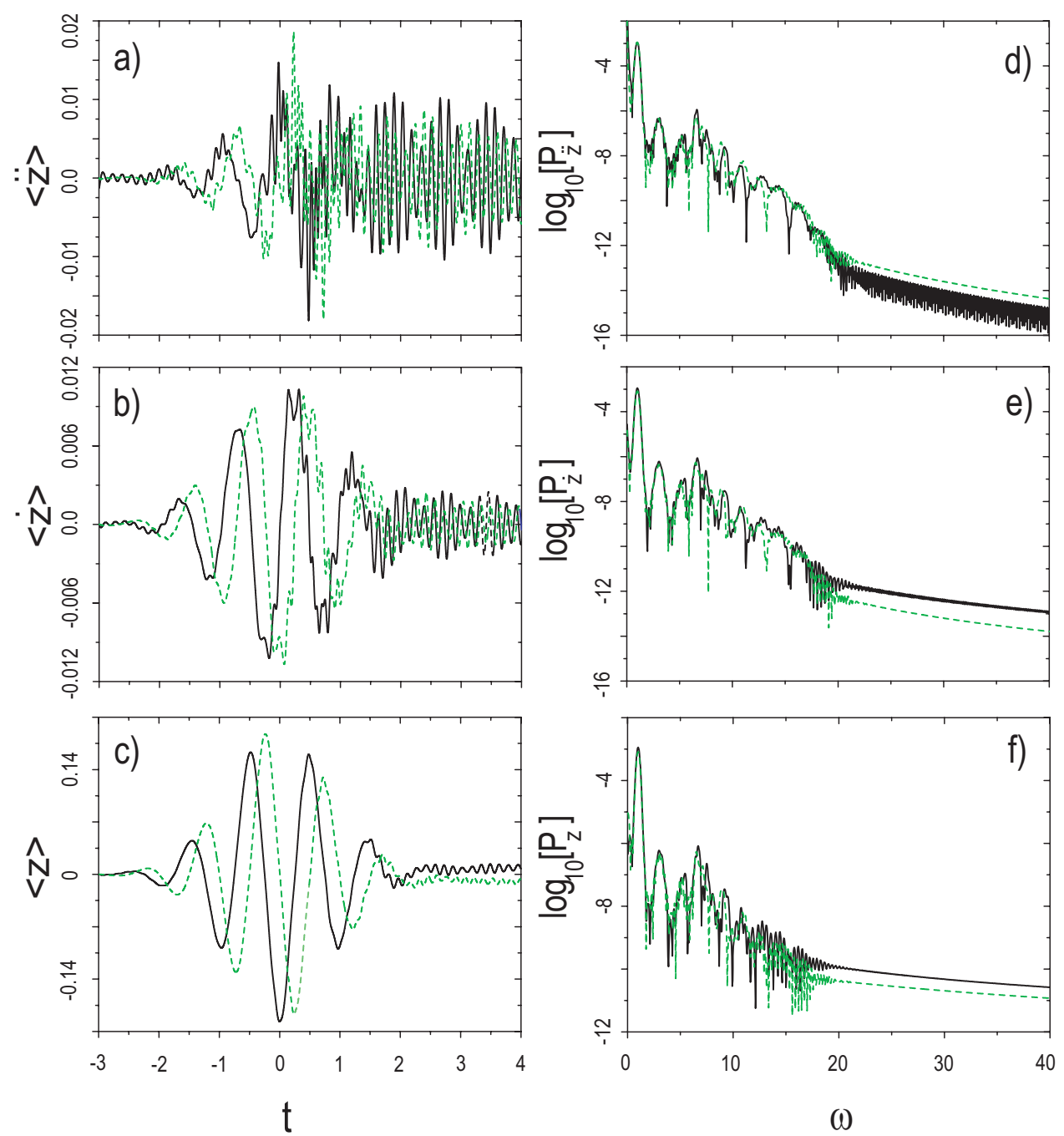

FIG. 3. (Color online) Same as Fig. 2, except for the pulse of nominal duration $6 \tau, t_{i}=-3 \tau$ and $t_{f}$ determined as described in Fig. 2 , such that actual duration is slightly longer than $6 \tau$. tions (2.29) and (2.31) imply that $N_{m}=15$ for $\eta=0$ compared with $N_{m}=13$ for $\eta=-\pi / 2$. The harmonic spectra plotted in Fig. 4 bear this out. For the purpose of this paper, however, the most important conclusion is that for the weak pulse $\left(I_{0}<I_{b}\right.$, the domain of tunneling ionization) the harmonic spectra for all three forms are in agreement, and this is mainly a consequence of the nearly vanishing mean dipole velocity and also rather small shifts of the mean dipole moment at the end of the laser pulse.

We now present results for the series of strong pulses $\left(I_{0}=10^{15} \mathrm{~W} \mathrm{~cm}^{-2}>I_{b}\right)$, starting with the longest $[T=16 \tau$, $\mathrm{FWHM}=9.33$ fs, $\eta=0,-\pi / 2$ (see Fig. 5)]. We note first that the influence of $\eta$ is not very pronounced, for the same reasons as in the case of the longest weak pulse (see Fig. 2). Second, we observe that the amplitudes of the oscillations in $\langle z(t)\rangle,\langle\dot{z}(t)\rangle$, and $\langle\ddot{z}(t)\rangle$ are much greater than for the corresponding weak pulses. The high electric field associated with the strong pulse induces sudden large-amplitude movement in $\langle z(t)\rangle$ [see Fig. 5(c)], not from the very beginning of the pulse, but only rather close to its maximum intensity, when the effective potential-energy (Coulombic+electricdipole interactions) is suppressed below the energy of the initial $(1 s)$ state, so that the electron can escape over the barrier. The $\langle\dot{z}(t)\rangle$ and $\langle\ddot{z}(t)\rangle$ exhibit corresponding sudden large-amplitude oscillations, compared with those induced by the weak pulse. The much larger amplitudes of $\langle z(t)\rangle$, $\langle\dot{z}(t)\rangle$, and $\langle\ddot{z}(t)\rangle$ naturally correlate with large increases in the magnitudes of the harmonic spectra. Another consequence of the higher intensity of the strong pulse is a dramatic increase from $N_{m}=15$ for the weak pulse to $N_{m}=70$ for the strong pulse.

Regardless of the substantial influence of intensity evident in Fig. 5, the most important conclusion, for the present purpose, is that $P_{\dot{z}}(\omega)$ and $P_{\ddot{z}}(\omega)$ are again very similar, at least for the domain of the harmonics below $N_{m}$. This can again be explained by Eq. (2.23) (i.e., the two spectra should agree for the ideal case $\left.\left\langle\dot{z}\left(t_{f}\right)\right\rangle=0\right)$. The apparent discrepancy between $P_{\dot{z}}(\omega)$ and $P_{\ddot{z}}(\omega)$ in the falloff domain may be a real effect, or a numerical artifact, for the following reason: close analysis reveals that it is almost impossible to realize numerically the constraint $\left\langle\dot{z}\left(t_{f}\right)\right\rangle=0$. In the present case, we could achieve $\left\langle\dot{z}\left(t_{f}\right)\right\rangle=8 \times 10^{-6}\left(e \hbar / a_{0} m_{e}\right)$, which is very small, yet still sufficiently large that the two $\left\langle\dot{z}\left(t_{f}\right)\right\rangle$-dependent terms in Eq. (2.23) appear to be dominant, compared to $D_{\ddot{z}}(\omega)$, as $\omega$ enters the falloff domain. For numerical reasons it is therefore essentially impossible to decide whether $P_{\dot{z}}(\omega)$ should also exhibit a falloff [as implied by Eq. (2.23) for the ideal case $\left.\left\langle\dot{z}\left(t_{f}\right)\right\rangle=0\right]$ for the case where $\left\langle\dot{z}\left(t_{f}\right)\right\rangle$ is very small but still not zero. We demonstrate below for the case of the shortest strong pulse that the apparent discrepancy between $P_{\ddot{z}}(\omega)$ 

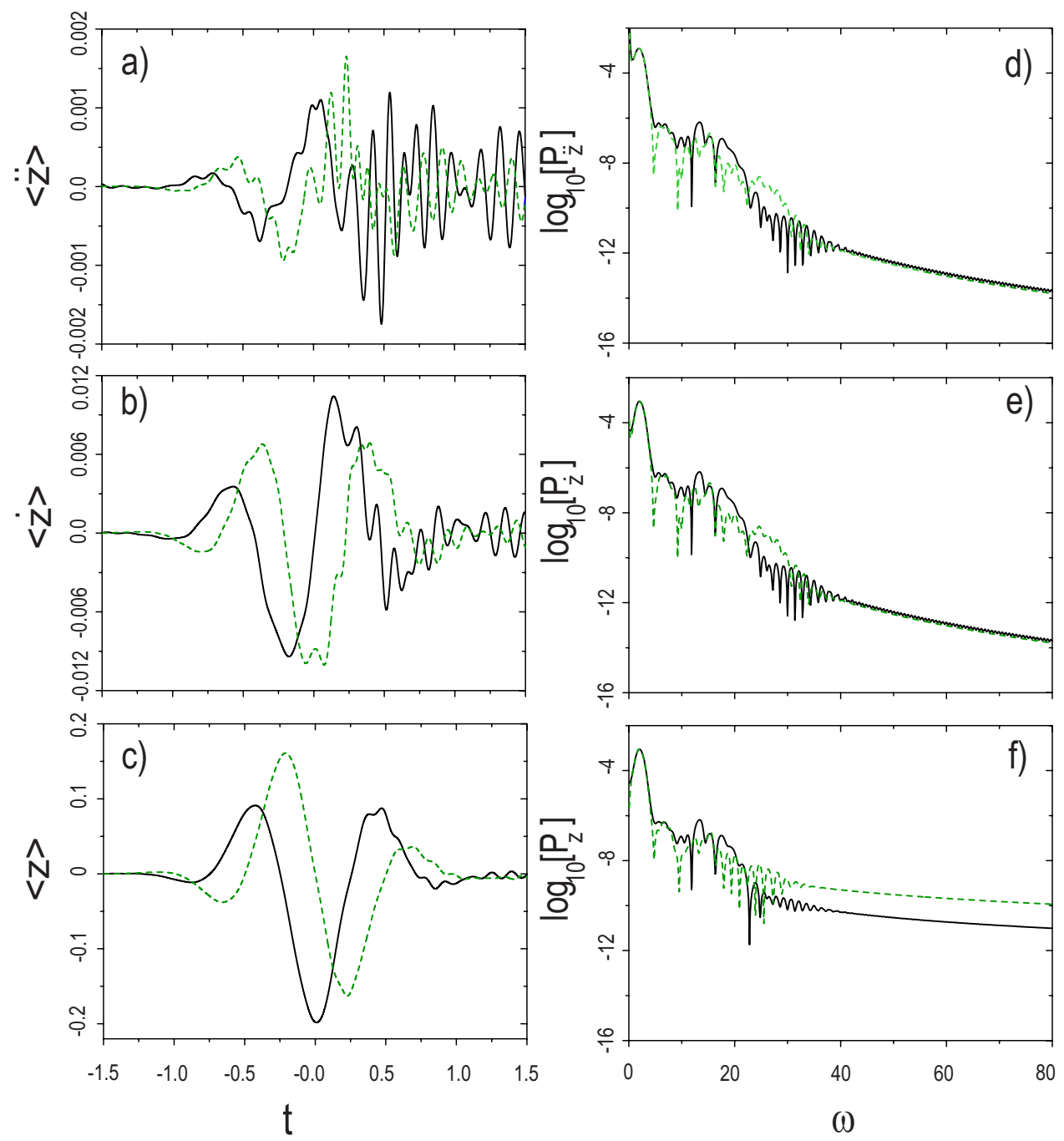

FIG. 4. (Color online) Same as Fig. 2, except for pulse of nominal duration $3 \tau ; t_{i}=-1.5 \tau$ and $t_{f}$ determined as described in Fig. 2, such that actual duration is slightly longer than $3 \tau$. and $P_{\dot{z}}(\omega)$ can be resolved. We note, however, that $P_{z}(\omega)$ is definitely different from either $P_{\dot{z}}(\omega)$ or $P_{\ddot{z}}(\omega)$ in almost all domains. This is a consequence of the rather large deviations of the limit contribution $\left\langle z\left(t_{f}\right)\right\rangle$ from zero [see Eq. (2.21)]. Thus, the plots of Figs. 1(a) and 5 suggest an important hypothesis: the HPNS [20] should be derivable from either the dipole-velocity or dipole-acceleration forms in the domains $n<N_{m}$, but not from the dipole form.

Results for the next shorter strong pulse $(T=6 \tau$, FWHM $=3.99 \mathrm{fs}$ ) are documented in Fig. 6, where it can be seen that $\eta$ has a heavy impact on $\left\langle z\left(t_{f}\right)\right\rangle$. This is also reflected in $\left\langle\dot{z}\left(t_{f}\right)\right\rangle$, which oscillates with small amplitude about zero for $\eta=-\pi / 2$, but about a negative value for $\eta=0$. Therefore, $P_{\dot{z}}(\omega)$ depends markedly on $\eta$. A prominent qualitative difference is that for $\eta=-\pi / 2, P_{\dot{z}}(\omega)$ should fall off for $n$ $>N_{m}$, whereas for $\eta=0$ it should not. At the same time the magnitude of $P_{\dot{z}}(\omega)$ for the higher harmonics is greater for $\eta=0$ than for $\eta=-\pi / 2$. This observation supports a working hypothesis: the previous finding of no falloff for the longer pulse [see Fig.5(e)] may also be a real effect. Moreover, the application of Eq. (2.23) for $\eta=-\pi / 2$, with $\left\langle\dot{z}\left(t_{f}\right)\right\rangle \approx 0$, implies that $P_{\dot{z}}(\omega)$ and $P_{\ddot{z}}(\omega)$ should be similar, whereas for $\eta$ $=0$, with $\left\langle\dot{z}\left(t_{f}\right)\right\rangle \neq 0, P_{\dot{z}}(\omega)$ and $P_{\ddot{z}}(\omega)$ should differ [compare Figs. 6(d) and 6(e)]. We note again that $P_{z}(\omega)$ differs drastically from both $P_{\dot{z}}(\omega)$ and $P_{\ddot{z}}(\omega)$.
Results for the shortest strong pulse $(T=3 \tau$, FWHM $=2.05 \mathrm{fs}$ ) are displayed in Fig. 7, where it is clear that the radically different asymptotic behavior of $\langle z(t)\rangle$ for $\eta=0$ and $\eta=-\pi / 2$ results in $\left\langle\dot{z}\left(t_{f}\right)\right\rangle \neq 0$. It follows that $P_{\dot{z}}(\omega)$ and $P_{\ddot{z}}(\omega)$ differ substantially for the two phases [see Figs. 7(d) and $7(\mathrm{e})]$. Most prominent is that $P_{\dot{z}}(\omega) \gg P_{\ddot{z}}(\omega)$. Moreover, $P_{\ddot{z}}(\omega)$ exhibits the expected falloff for both CEPs, with different cutoffs close to $N_{m}=70$ for $\eta=0$ versus $N_{m}=52$ for $\eta=-\pi / 2$, in accord with Eq. (2.31). In sharp contrast, $P_{\dot{z}}(\omega)$ exhibits no such falloff.

It is instructive to analyze the results in Fig. 7 in terms of the two-step quasistatic tunneling model [38]. In the first step the $\mathrm{H}$ atom is ionized, predominantly during a short time about the maximum of the electric-field amplitude, where the carrier envelope remains approximately constant. It is assumed that the electron is liberated at $t=t_{0}$ with dipole moment $\mathbf{r}\left(t_{0}\right)$ and dipole velocity $\dot{\mathbf{r}}\left(t_{0}\right)=0$ [25]. In the second step the electron moves classically under the action of only the field of the pulse. Its Coulombic attraction to the proton is ignored. Newton's second law then yields

$$
\begin{gathered}
z(t)=z\left(t_{0}\right)+z_{o s c}+\dot{z}_{d}\left(t_{0}\right)\left[t-t_{0}\right], \\
\dot{z}(t)=\dot{z}_{d}\left(t_{0}\right)-\left(E_{0} / \omega_{0}\right) \sin \left(\omega_{0} t+\eta\right),
\end{gathered}
$$

where 

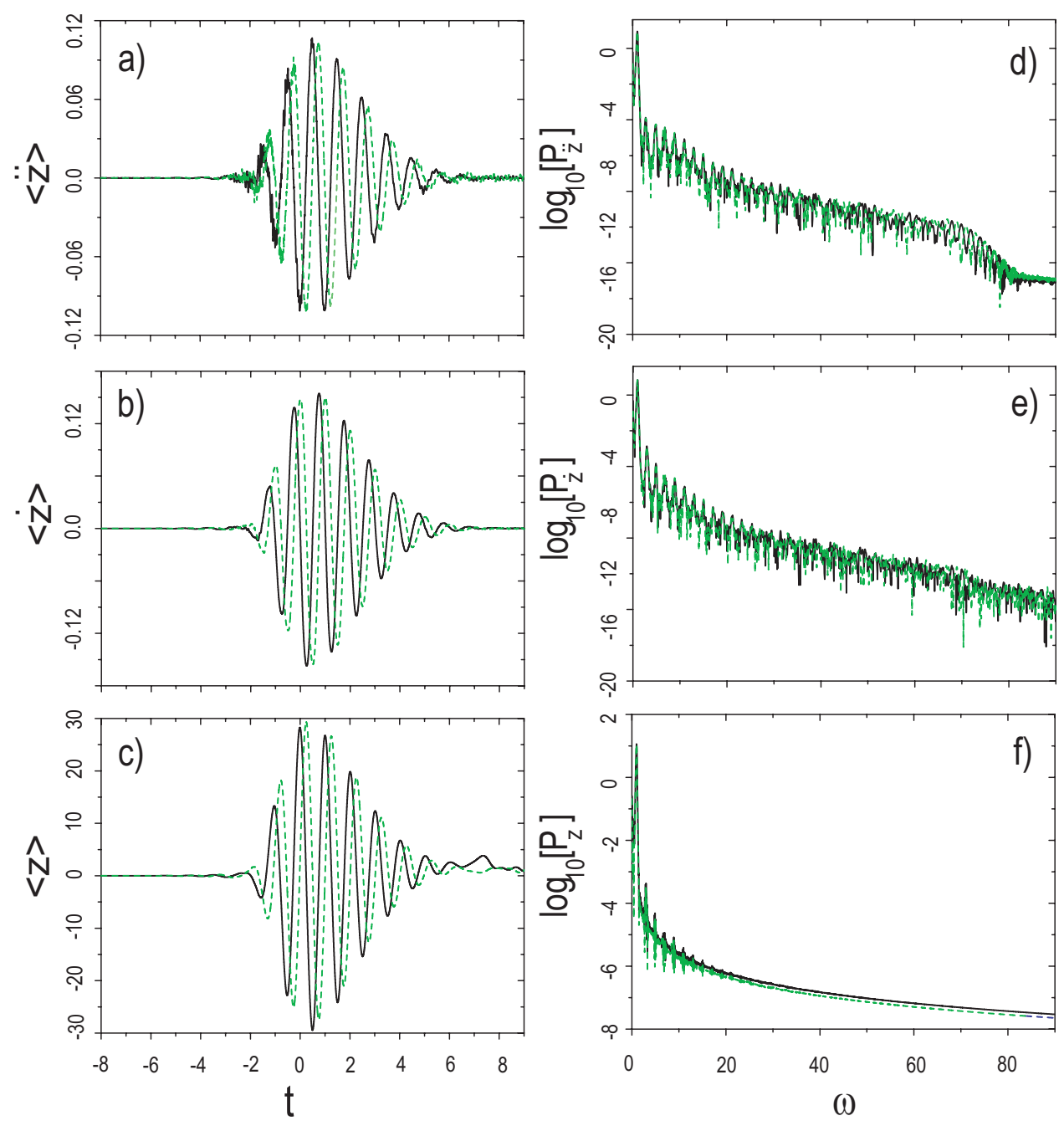

FIG. 5. (Color online) Same as Fig. 2, except for strong pulse $\left(I_{0}\right.$ $\left.=10^{15} \mathrm{~W} \mathrm{~cm}^{-2}, E_{0}=0.119 E_{h} / a_{0} e\right)$ of nominal duration $16 \tau$. $t_{i}=-8 \tau$ and $t_{f}$ determined as described in Fig. 2, such that actual duration is slightly longer than $16 \tau$.

$$
\begin{gathered}
z_{o s c} \equiv\left(E_{0} / \omega_{0}^{2}\right)\left[\cos \left(\omega_{0} t+\eta\right)-\cos \left(\omega_{0} t_{0}+\eta\right)\right] \\
\dot{z}_{d}\left(t_{0}\right) \equiv\left(E_{0} / \omega_{0}\right) \sin \left(\omega_{0} t_{0}+\eta\right)
\end{gathered}
$$

At long times the last term on the right side of Eq. (4.1a) dominates. The electron drifts away from the proton with approximately constant velocity $\dot{z}_{d}\left(t_{0}\right)$. From Eq. (4.2b) it follows that $\dot{z}_{d}\left(-t_{0}\right)=-\dot{z}_{d}\left(t_{0}\right)$ when $\eta=0$ and $\dot{z}_{d}\left(-t_{0}\right)=\dot{z}_{d}\left(t_{0}\right)$ when $\eta=-\pi / 2$. Hence, since the ionization rate is approximately symmetric in time about the maximum, the average drift (dipole) velocity of the electron should vanish when $\eta$ $=0$, whereas it should be either negative or positive when $\eta=-\pi / 2$. In other words, when $\eta=0$, electrons are emitted in the positive and negative $z$ directions with equal likelihood, whereas when $\eta=-\pi / 2$, they are emitted preferentially in either the negative or the positive $z$ direction. The two CEPs $\eta=0$ and $\eta=-\pi / 2$, respectively, correspond to symmetric and asymmetric photoelectron kinetic-energy spectra [38]. The plots in Figs. 7(b) and 7(c) are roughly in accord with the model. Although $\left\langle\dot{z}\left(t_{f}\right)\right\rangle \neq 0$ when $\eta=0$, it is much smaller than $\left\langle\dot{z}\left(t_{f}\right)\right\rangle$ for $\eta=-\pi / 2$. We note that since the (mean) drift, or postpulse, velocity is constant, the (mean) postpulse acceleration vanishes [see Fig. 7(a)].
The predicted harmonic spectra depend on the length of the period over which photons are collected. Until now we have regarded this period to be the same as the duration $T$ of the pulse. We now consider the extreme case of collecting photons forever (i.e., from $t_{i}$ to $\infty$ ). For this purpose we employ a numerical trick: we define $t_{f}$ not by $\left\langle\dot{z}\left(t_{f}\right)\right\rangle=0$, as for the results shown in Figs. $2-5$, but rather by choosing $t_{f}$ slightly greater than $-t_{i}$ so that $\left\langle\ddot{z}\left(t_{f}\right)\right\rangle=0$. Furthermore, we set $\langle\ddot{z}(t)\rangle=0$ for times $t>t_{f}$, assuming that there are no significant effects of accelerations after the passage of the pulse. Thus, in the FT [see Eq. (2.8)] the upper limit can be extended to $\infty$ for the acceleration form, simply because there is no contribution beyond $t=t_{f}$. We refer to the acceleration form of the spectrum thus calculated as the infinite-time form, denoted by $P_{\ddot{z}}^{\inf }(\omega)$. It is numerically advantageous since it can be calculated rigorously from the numerically generated $\langle\ddot{z}(t)\rangle$ and then used in Eq. (2.23) to compute a corresponding infinite-time dipole-velocity spectrum $P_{\dot{z}}^{\mathrm{inf}}(\omega)$. A comparison of $P_{\dot{z}}(\omega)$ and $P_{\dot{z}}^{\mathrm{inf}}(\omega)$ is made in Fig. 8 for $\eta$ $=0$ [see Fig. 7(b)]. The agreement is nearly quantitative. Similar results are obtained for the case represented by Fig. 6 . We conclude that most of the photons are produced during $T$ and that collecting photons for longer times will not significantly alter the harmonic spectra. 

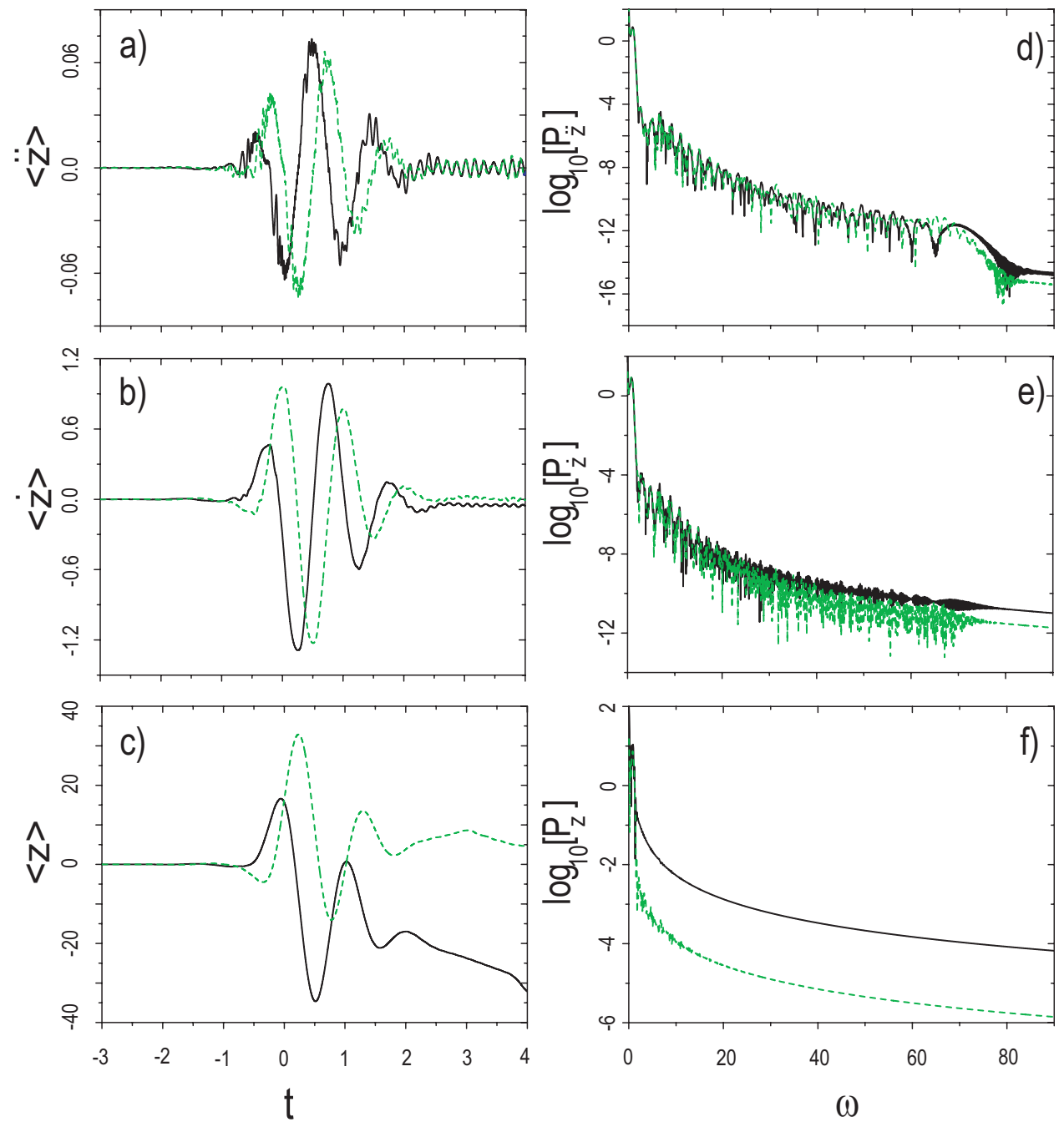

FIG. 6. (Color online) Same as Fig. 5, except nominal duration is $6 \tau . t_{i}=-3 \tau ; t_{f}$ chosen close to, but slightly larger than $-t_{i}$, such that $\left\langle\ddot{z}\left(t_{f}\right)\right\rangle=0$. Actual duration is slightly longer than $6 \tau$.
Finally, we note that harmonic spectra corresponding to the long pulses (see Figs. 2 and 5) manifest the "rule" that only odd harmonics appear in the domain $n<N_{m}$ [33], in stark contrast to the spectra produced by the shortest pulses (Figs. 4 and 7), which disobey the rule. The reason for this breakdown is that the single carrier frequency $\omega_{0}$ dominates the long pulses, whereas many frequencies effectively contribute to the short ones. In the extreme limit of ultrashort (few-attosecond) pulses, the distinction between odd and even harmonics disappears and the HHG spectrum becomes continuous $[24,39]$. This is also reflected in the strong asymmetry seen in simulations of photoelectron kinetic-energy spectra [40].

\section{CONCLUSION}

In this paper we have presented comparisons of three forms of the harmonic spectrum, based on dipole, dipole velocity, and dipole acceleration, by solving the Schrödinger equation numerically for the hydrogen atom interacting with a laser pulse whose electric field is specified by $E(t)$ $=E_{0} f(t) \cos \left(\omega_{0} t+\eta\right)$, where $f(t)=\exp \left(-t^{2} / \delta^{2}\right)$. We have examined the influence of intensity $I_{0}$ (proportional to $E_{0}^{2}$ ), duration $T$ (proportional to $\delta$ ), and the phase $\eta$ of the carrier envelope. We have considered two sets of pulses: (1) "weak" $\left(I_{0}=10^{14} \mathrm{~W} \mathrm{~cm}^{-2}<I_{b}=1.4 \times 10^{14} \mathrm{~W} \mathrm{~cm}^{-2}\right.$, the threshold intensity for over-barrier ionization) with durations $T$ between 3 and 16 optical cycles; (2) "strong" $\left(I_{0}=10^{15} \mathrm{~W} \mathrm{~cm}^{-2}>I_{b}\right)$ with the same durations. The carrier frequency $\omega_{0}$ corresponds to the wavelength $800 \mathrm{~nm}$ for all pulses.

The principal finding is that for long, weak pulses the three forms of the harmonic spectrum are in good agreement. This is so because the limit contributions by which the forms differ (except for scalings by powers of frequency) in general, according to Eqs. (2.21) and (2.23), are negligible when the pulse is long and weak. This confirms previous findings by Telnov and Chu [21] for HHG rates based on weak, periodic, or continuous-wave fields. On the other hand, as the pulse becomes stronger and shorter, the limit contributions increase gradually and the three spectral forms tend to diverge.

Following the passage of a short (few-cycle), intense pulse that ionizes the $\mathrm{H}$ atom, $\langle z(t)\rangle$ increases without bound (corresponding to unidirectional ionization [41,42]) while $\langle\dot{z}(t)\rangle$ oscillates about a nonzero value, whereas $\langle\ddot{z}(t)\rangle$ decays to zero. By a mathematical artifice we can exploit this behavior to reconcile the divergent forms. In particular, for the strongest, shortest pulse studied here, we demonstrate explicitly how the infinite-time dipole-velocity spectrum $P_{\dot{z}}^{\text {inf }}(\omega)$ 

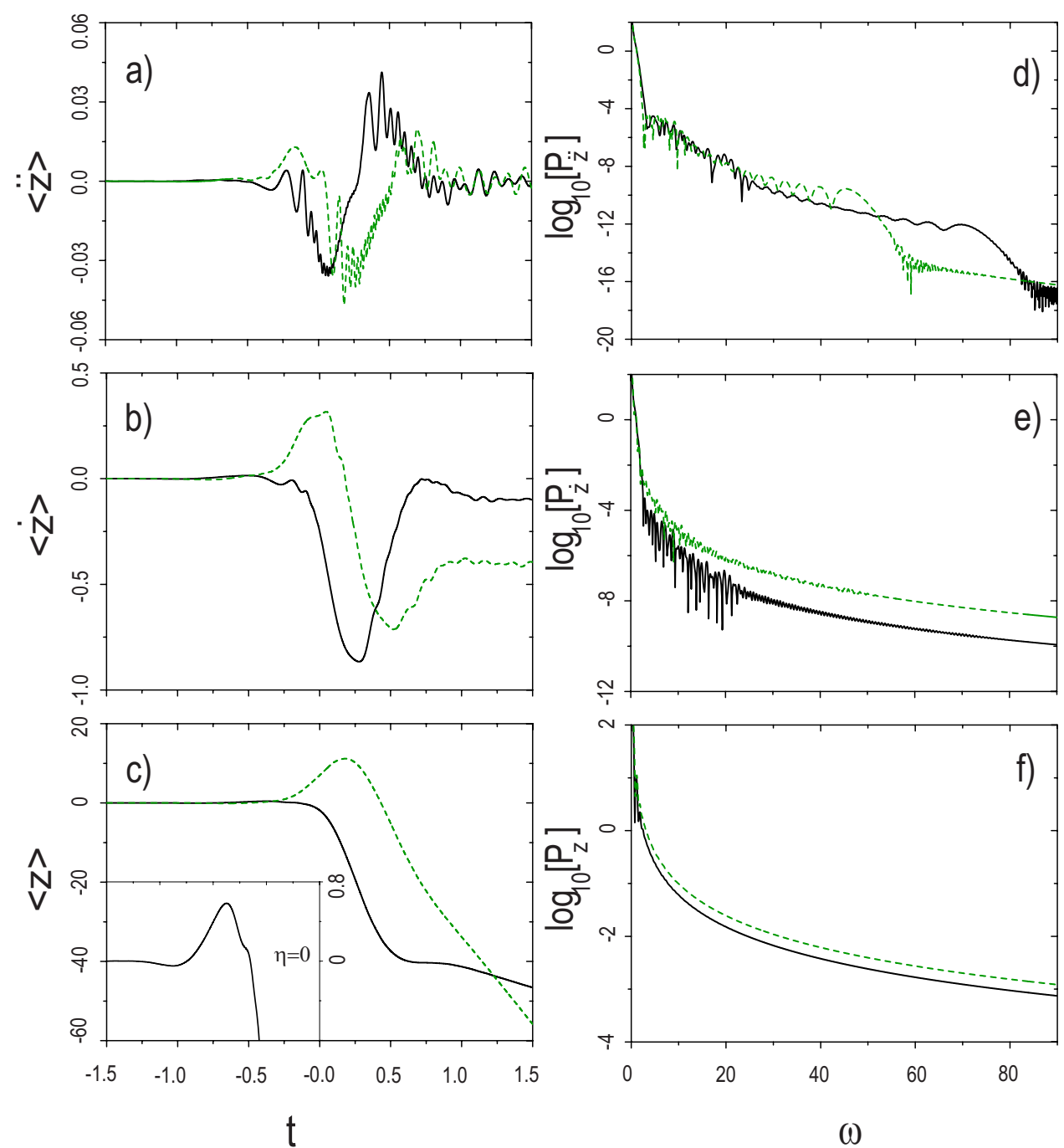
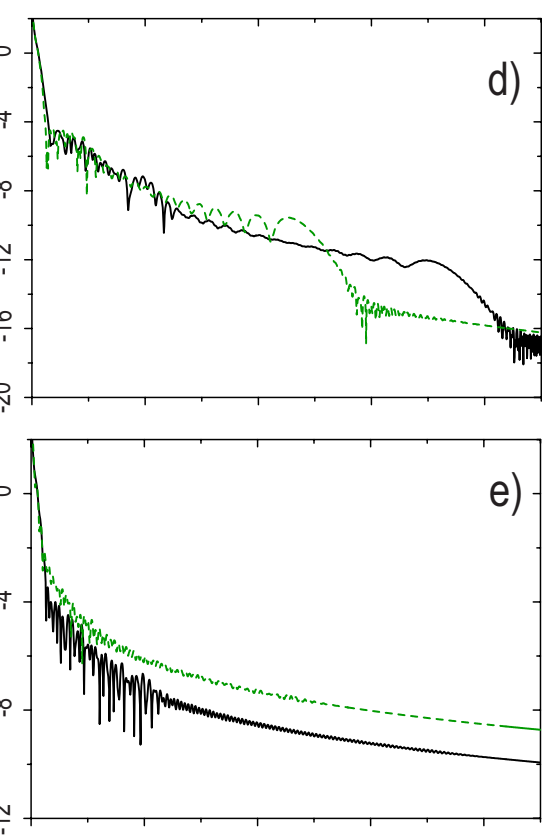

$\omega$
FIG. 7. (Color online) Same as Fig. 5, except nominal duration is $3 \tau . t_{i}=-1.5 \tau$ and $t_{f}$ determined as described in Fig. 6, such that actual duration is slightly longer than $3 \tau$. The inset in (c) displays $\langle z(t)\rangle$ on fine scale. can be computed from the infinite-time acceleration spectrum $P_{\ddot{z}}^{\inf }(\omega)$. $P_{\dot{z}}^{\text {inf }}(\omega)$ is shown to agree almost quantitatively with the spectrum $P_{\dot{z}}(\omega)$ computed for the finite time $T=t_{f}$ $-t_{i}$, namely, the pulse duration. Apparently most of the photons that contribute to the spectrum are produced during the passage of the pulse. Hence, even though the (idealized) experimental spectrum (HPNS) [20] is expressed in terms of the dipole-velocity form, the acceleration form affords a convenient numerical route to the HPNS.

The relations in Eqs. (2.21) and (2.23) are especially useful for calculating and interpreting harmonic spectra currently being generated with intense, few-to single-cycle laser pulses $[23,24]$. We emphasize the extreme sensitivity of spectra to the CEP $\eta$ for intense, ultrashort pulses, which was also observed in previous simulations [43]. One needs the precise formulation represented by Eqs. (2.21) and (2.23) in order to handle such pulses, which give rise to nonvanishing final (mean) dipole moment $\left\langle z\left(t_{f}\right)\right\rangle$ and (mean) dipole velocity $\left\langle\dot{z}\left(t_{f}\right)\right\rangle$. These postpulse "residues" have a critical impact on the symmetry of photoelectron kinetic-energy spectra (a signature of the CEP $[27,38,40,41,44])$, as well as on the "parity" of the harmonics (i.e., both odd and even harmonics appear in the spectra [39]). It is particularly noteworthy that, since the postpulse (mean) dipole velocity is constant and the (mean) dipole acceleration vanishes, the acceleration form of the harmonic spectrum provides a superior computational pathway to the number of harmonic photons. Finally, we note that our results are also relevant to the analysis of

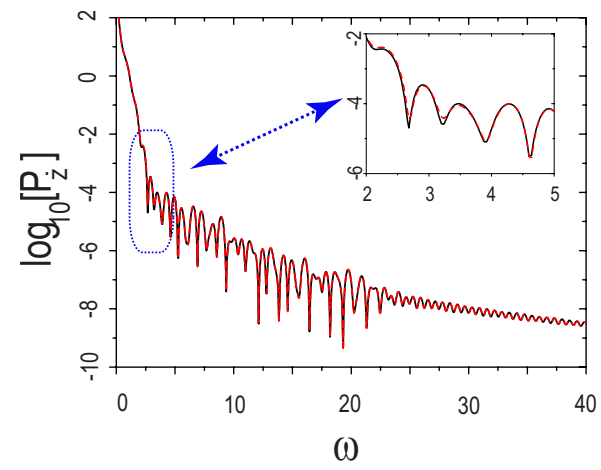

FIG. 8. (Color online) Comparison of infinite-time spectrum $P_{z}^{\inf }(\omega)$ [in atomic units of $\left(e a_{0}\right)^{2}$, as a function of frequency in units of the carrier frequency $\omega_{0}$ ] (dashed line, computed as described in text) with finite-time spectrum $P_{\dot{z}}(\omega)$ [solid line, displayed in Fig. 7(e)] for shortest, strong pulse with $\eta=0$. 
orientation-dependent molecular high-harmonic spectra in orbital tomography [17], which leads to confirmation of the three-step recollision model [25] for molecules [7].

\section{ACKNOWLEDGMENTS}

The authors are grateful to Ingo Barth (Berlin) for diligently checking various formulas and to Robert Gordesch (Berlin) for checking selected numerical results. They are also thankful to Professor Paul Corkum (Ottawa) for clarifying the HHG experiment. A.D.B. and J.M. thank the Alexander von Humboldt Foundation for financial support of A.D.B. J.M. also gratefully acknowledges the support of the Deutsche Forschungsgemeinschaft (through Project No. Sfb 450-TP C1) and of the Fonds der Chemischen Industrie. D.J.D. thanks the Freie Universität Berlin for financial support for several visits.

\section{APPENDIX A}

For the convenience of the reader in following the developments in Sec. II, we summarize here relevant properties of the (spherical) Legendre polynomials $P_{l}(\cos \theta)$ [45].

$$
\begin{aligned}
& \int_{0}^{\pi} d \theta \sin \theta P_{l}(\cos \theta) P_{k}(\cos \theta) \\
& =\int_{-1}^{1} d x P_{l}(x) P_{k}(x)=2 \delta_{l k} /(2 l+1), \\
& \int_{0}^{\pi} d \theta \sin \theta P_{l}(\cos \theta) \cos \theta P_{k}(\cos \theta) \\
& =\int_{-1}^{1} d x P_{l}(x) x P_{k}(x) \\
& =2 l \delta_{l, k+1} /[(2 l+1)(2 l-1)] \\
& +2 k \delta_{k, l+1} /[(2 k+1)(2 k-1)] \\
& \left(1-x^{2}\right) \frac{d P_{k}(x)}{d x}=-k x P_{k}(x)+k P_{k-1}(x) .
\end{aligned}
$$

\section{APPENDIX B}

Equation (2.14) can be rewritten as

$$
\begin{aligned}
\langle\dot{z}(t)\rangle= & -i \sum_{l} \sum_{k}\left\{\int_{0}^{\infty} d r r^{2} R_{l}^{*}(r, t) \frac{\partial R_{k}(r, t)}{\partial r}\right. \\
& \times \int_{l} d \Omega Y_{l 0}^{*}(\theta, \phi) \cos \theta Y_{k 0}(\theta, \phi) \\
& -\int_{0}^{\infty} d r r R_{l}^{*}(r, t) \frac{R_{k}(r, t)}{r} \\
& \left.\times \int d \Omega Y_{l 0}^{*}(\theta, \phi) \sin \theta \frac{\partial Y_{k 0}(\theta, \phi)}{\partial \theta}\right\} .
\end{aligned}
$$

Substituting Eq. (2.4) into Eq. (B1) and making the change of variables $x=\cos \theta$, we get

$$
\begin{aligned}
\langle\dot{z}(t)\rangle= & -\frac{i}{2} \sum_{l} \sum_{k}[(2 l+1)(2 k+1)]^{1 / 2} \\
& \times\left\{\int_{0}^{\infty} d r r^{2} R_{l}^{*}(r, t) \frac{\partial R_{k}(r, t)}{\partial r} \int_{-1}^{1} d x P_{l}(x) x P_{k}(x)\right. \\
& \left.+\int_{0}^{\infty} d r r R_{l}^{*}(r, t) R_{k}(r, t) \int_{-1}^{1} d x P_{l}(x)\left(1-x^{2}\right) \frac{d P_{k}(x)}{d x}\right\},
\end{aligned}
$$

where we have performed the integration on the azimuthal angle. Utilizing the differential relation (A3), we can rewrite Eq. (B2) as

$$
\begin{aligned}
\langle\dot{z}(t)\rangle= & -\frac{i}{2} \sum_{l} \sum_{k}[(2 l+1)(2 k+1)]^{1 / 2} \\
& \times\left\{\int_{0}^{\infty} d r r^{2} R_{l}^{*}(r, t) \frac{\partial R_{k}(r, t)}{\partial r} \int_{-1}^{1} d x P_{l}(x) x P_{k}(x)\right. \\
& +\int_{0}^{\infty} d r r R_{l}^{*}(r, t) R_{k}(r, t) \\
& \left.\times\left[-k \int_{-1}^{1} d x P_{l}(x) x P_{k}(x)+k \int_{-1}^{1} d x P_{l}(x) P_{k-1}(x)\right]\right\} .
\end{aligned}
$$

Inserting the relations in Eqs. (A1) and (A2) into Eq. (B3) yields

$$
\begin{aligned}
\langle\dot{z}(t)\rangle= & -\frac{i}{2} \sum_{l} \sum_{k}[(2 l+1)(2 k+1)]^{1 / 2}\left\{\int_{0}^{\infty} d r r^{2} R_{l}^{*} \frac{\partial R_{k}}{\partial r}\right. \\
& \times\left(2 l \delta_{l, k+1} /\right. \\
& {\left.[(2 l+1)(2 l-1)]+2 k \delta_{k, l+1} /[(2 k+1)(2 k-1)]\right) } \\
& +\int_{0}^{\infty} d r r R_{l}^{*} R_{k}\left[-k\left(2 l \delta_{l, k+1} /[(2 l+1)(2 l-1)]\right.\right. \\
& \left.\left.\left.+2 k \delta_{k, l+1} /[(2 k+1)(2 k-1)]\right)+k 2 \delta_{l, k-1} /(2 l+1)\right]\right\},
\end{aligned}
$$

which can be expanded in detail as 


$$
\begin{aligned}
\langle\dot{z}(t)\rangle= & -\frac{i}{2}\left\{\sum_{l} \sum_{k}[(2 l+1)(2 k+1)]^{1 / 2} \int_{0}^{\infty} d r r^{2} R_{l}^{*} \frac{\partial R_{k}}{\partial r}\left(\frac{2 l \delta_{l, k+1}}{[(2 l+1)(2 l-1)]}\right)+\sum_{l} \sum_{k}[(2 l+1)(2 k+1)]^{1 / 2}\right. \\
& \times \int_{0}^{\infty} d r r^{2} R_{l}^{*} \frac{\partial R_{k}}{\partial r}\left(\frac{2 k \delta_{k, l+1}}{[(2 k+1)(2 k-1)]}\right)-\sum_{l} \sum_{k}[(2 l+1)(2 k+1)]^{1 / 2} \int_{0}^{\infty} d r r R_{l}^{*} R_{k}\left(\frac{2 k l \delta_{l, k+1}}{[(2 l+1)(2 l-1)]}\right) \\
& \left.-\sum_{l} \sum_{k}[(2 l+1)(2 k+1)]^{1 / 2} \int_{0}^{\infty} d r r R_{l}^{*} R_{k}\left(\frac{2 k^{2} \delta_{k, l+1}}{[(2 k+1)(2 k-1)]}\right)+\sum_{l} \sum_{k}[(2 l+1)(2 k+1)]^{1 / 2} \int_{0}^{\infty} d r r R_{l}^{*} R_{k}\left(\frac{2 k \delta_{l, k-1}}{(2 l+1)}\right)\right\} .
\end{aligned}
$$

Carrying out the summations on $l$ in the first and third terms and on $k$ in the second, fourth, and fifth terms, respectively, in Eq. (B5), we obtain

$$
\begin{aligned}
\langle\dot{z}(t)\rangle= & -\frac{i}{2}\left\{\sum_{k} \frac{[(2 k+1)(2 k+3)]^{1 / 2}}{(2 k+1)(2 k+3)} 2(k+1) \int_{0}^{\infty} d r r^{2} R_{k+1}^{*} \frac{\partial R_{k}}{\partial r}+\sum_{l} \frac{[(2 l+1)(2 l+3)]^{1 / 2}}{(2 l+1)(2 l+3)} 2(l+1)\right. \\
& \times \int_{0}^{\infty} d r r^{2} R_{l}^{*} \frac{\partial R_{l+1}}{\partial r}-\sum_{k} \frac{[(2 k+1)(2 k+3)]^{1 / 2}}{(2 k+1)(2 k+3)} 2 k(k+1) \int_{0}^{\infty} d r r R_{k+1}^{*} R_{k}-\sum_{l} \frac{[(2 l+1)(2 l+3)]^{1 / 2}}{(2 l+1)(2 l+3)} 2(l+1)^{2} \\
& \left.\times \int_{0}^{\infty} d r r R_{l}^{*} R_{l+1}+\sum_{l} \frac{[(2 l+1)(2 l+3)]^{1 / 2}}{(2 l+1)(2 l+3)} 2(l+1)(2 l+3) \int_{0}^{\infty} d r r R_{l}^{*} R_{l+1}\right\} .
\end{aligned}
$$

By redefining dummy indices and combining terms we can simplify Eq. (B6) to

$$
\begin{aligned}
\langle\dot{z}(t)\rangle= & -i\left\{\sum_{l} \frac{(l+1)}{[(2 l+1)(2 l+3)]^{1 / 2}}\left[\int_{0}^{\infty} d r r^{2} R_{l}^{*} \frac{\partial R_{l+1}}{\partial r}+\int_{0}^{\infty} d r r^{2} R_{l+1}^{*} \frac{\partial R_{l}}{\partial r}\right]\right. \\
& \left.-\sum_{l} \frac{l(l+1)}{[(2 l+1)(2 l+3)]^{1 / 2}} \int_{0}^{\infty} d r r R_{l} R_{l+1}^{*}+\sum_{l} \frac{(l+1)(l+2)}{[(2 l+1)(2 l+3)]^{1 / 2}} \int_{0}^{\infty} d r r R_{l}^{*} R_{l+1}\right\} .
\end{aligned}
$$

Now consider the integral

$$
I \equiv \int_{0}^{\infty} d r r^{2} R_{l}^{*} \frac{d R_{l+1}}{d r}, \quad t \text { fixed }
$$

which appears in the first term on the right side of Eq. (B7). Integration by parts yields

$$
I=\left.r^{2} R_{l}^{*} R_{l+1}\right|_{0} ^{\infty}-\int_{0}^{\infty} d r\left[2 r R_{l}^{*}+r^{2} \frac{d R_{l}^{*}}{d r}\right] R_{l+1} .
$$

The first term on the right side of Eq. (B9) vanishes by virtue of the boundary conditions. Substitution of Eq. (B9) into Eq. (B7) gives

$$
\begin{aligned}
\langle\dot{z}(t)\rangle= & -i\left\{\sum_{l} \frac{(l+1)}{[(2 l+1)(2 l+3)]^{1 / 2}}\left[-2 \int_{0}^{\infty} d r r R_{l}^{*} R_{l+1}-\int_{0}^{\infty} d r r^{2} R_{l+1} \frac{\partial R_{l}^{*}}{\partial r}+\int_{0}^{\infty} d r r^{2} R_{l+1}^{*} \frac{\partial R_{l}}{\partial r}\right]\right. \\
& \left.-\sum_{l} \frac{l(l+1)}{[(2 l+1)(2 l+3)]^{1 / 2}} \int_{0}^{\infty} d r r R_{l} R_{l+1}^{*}+\sum_{l} \frac{l(l+1)+2(l+1)}{[(2 l+1)(2 l+3)]^{1 / 2}} \int_{0}^{\infty} d r r R_{l}^{*} R_{l+1}\right\} .
\end{aligned}
$$

Combining like terms in Eq. (B10), we obtain

$$
\begin{aligned}
\langle\dot{z}(t)\rangle= & -i\left\{\sum_{l} \frac{(l+1)}{[(2 l+1)(2 l+3)]^{1 / 2}}\left[\int_{0}^{\infty} d r r^{2} \frac{\partial R_{l}}{\partial r} R_{l+1}^{*}-\int_{0}^{\infty} d r r^{2} \frac{\partial R_{l}^{*}}{\partial r} R_{l+1}\right]\right. \\
& \left.-\sum_{l} \frac{l(l+1)}{[(2 l+1)(2 l+3)]^{1 / 2}}\left[\int_{0}^{\infty} d r r R_{l} R_{l+1}^{*}-\int_{0}^{\infty} d r r R_{l}^{*} R_{l+1}\right]\right\} .
\end{aligned}
$$

Now observing that the pairs of terms in brackets are complex conjugates of each other, we can recast Eq. (B11) as 


$$
\begin{aligned}
\langle\dot{z}(t)\rangle & =-i\left\{\sum_{l} \frac{(l+1)}{[(2 l+1)(2 l+3)]^{1 / 2}} 2 i \operatorname{Im}\left[\int_{0}^{\infty} d r r^{2} \frac{\partial R_{l}}{\partial r} R_{l+1}^{*}\right]-\sum_{l} \frac{l(l+1)}{[(2 l+1)(2 l+3)]^{1 / 2}} 2 i \operatorname{Im}\left[\int_{0}^{\infty} d r r R_{l} R_{l+1}^{*}\right]\right\} \\
& =2 \sum_{l} \frac{(l+1)}{[(2 l+1)(2 l+3)]^{1 / 2}}\left\{\operatorname{Im}\left[\int_{0}^{\infty} d r r^{2} \frac{\partial R_{l}}{\partial r} R_{l+1}^{*}\right]-l \operatorname{Im}\left[\int_{0}^{\infty} d r r R_{l} R_{l+1}^{*}\right]\right\} .
\end{aligned}
$$

[1] T. Brabec and F. Krausz, Rev. Mod. Phys. 72, 545 (2000).

[2] P. B. Corkum and F. Krausz, Nat. Phys. 3, 381 (2007).

[3] S. Patchkovskii, Z. Zhao, T. Brabec, and D. M. Villeneuve, Phys. Rev. Lett. 97, 123003 (2006).

[4] B. Sundaram and P. W. Milonni, Phys. Rev. A 41, 6571 (1990).

[5] J. L. Krause, K. J. Schafer, and K. C. Kulander, Phys. Rev. Lett. 68, 3535 (1992).

[6] H. Xu, X. Tang, and P. Lambropoulos, Phys. Rev. A 46, R2225 (1992).

[7] M. Lewenstein, Ph. Balcou, M. Yu. Ivanov, A. L'Huillier, and P. B. Corkum, Phys. Rev. A 49, 2117 (1994).

[8] X.-M. Tong and S.-I. Chu, Chem. Phys. 217, 119 (1997).

[9] G. Lagmago Kamta and A. D. Bandrauk, Phys. Rev. A 71, 053407 (2005).

[10] J. Chen and S. G. Chen, Phys. Rev. A 75, 041402(R) (2007).

[11] A. D. Bandrauk, S. Barmaki, and G. Lagmago Kamta, in Progress in Ultrafast Intense Laser Science, edited by K. Yamanouchi (Springer, New York, 2007), Vol. 3.

[12] C. C. Chirilă and M. Lein, J. Mod. Opt. 54, 1039 (2007).

[13] K. Burnett, V. C. Reed, J. Cooper, and P. L. Knight, Phys. Rev. A 45, 3347 (1992).

[14] A. Sanpera, P. Jönsson, J. B. Watson, and K. Burnett, Phys. Rev. A 51, 3148 (1995).

[15] J. Prager, S. X. Hu, and C. H. Keitel, Phys. Rev. A 64, 045402 (2001).

[16] S. X. Hu and L. A. Collins, Phys. Rev. A 69, 033405 (2004).

[17] J. Itatani, J. Levesque, D. Zeidler, H. Niikura, H. Pépin, J. C. Kieffer, P. B. Corkum, and D. M. Villeneuve, Nature (London) 432, 867 (2004).

[18] M. Lein, J. Phys. B 40, R135 (2007).

[19] J. J. Carrera, X. M. Tong, and Shih-I. Chu, Phys. Rev. A 74, 023404 (2006).

[20] D. J. Diestler, Phys. Rev. A 78, 033814 (2008).

[21] D. A. Telnov and Shih-I. Chu, Phys. Rev. A 71, 013408 (2005).

[22] E. Lorin, S. Chelkowski, and A. D. Bandrauk, New J. Phys. 10, 025033 (2008).

[23] E. Goulielmakis, M. Schultze, M. Hofstetter, V. S. Yakovlev, J. Gagnon, M. Uiberacker, A. L. Aquila, E. M. Gullikson, D. T. Attwood, R. Kienberger, F. Krausz, and U. Kleineberg, Science 320, 1614 (2008).

[24] F. Lindner, M. G. Schätzel, H. Walther, A. Baltuška, E. Goul- ielmakis, F. Krausz, D. B. Milošević, D. Bauer, W. Becker, and G. G. Paulus, Phys. Rev. Lett. 95, 040401 (2005).

[25] P. B. Corkum, Phys. Rev. Lett. 71, 1994 (1993).

[26] See, for example, Y.-S. Huang and K.-H. Lu, Found. Phys. 38, 151 (2008).

[27] D. B. Milošević, G. G. Paulus, D. Bauer, and W. Becker, J. Phys. B 39, R203 (2006).

[28] W. H. Flygare, Molecular Structure and Dynamics (PrenticeHall, Englewood Cliffs, New Jersey, 1978), Chap. 1.

[29] L. V. Keldysh, Sov. Phys. JETP 20, 1307 (1965).

[30] See, for example, W. H. Louisell, Quantum Statistical Properties of Radiation (Wiley, New York, 1973), p. 64ff.

[31] M. D. Feit and J. A. Fleck, Jr., J. Chem. Phys. 78, 301 (1983).

[32] P. L. DeVries, J. Opt. Soc. Am. B 7, 517 (1990).

[33] J. L. Krause, K. J. Schafer, and K. C. Kulander, Phys. Rev. A 45, 4998 (1992).

[34] See, for example, S. Chelkowski and A. D. Bandrauk, Phys. Rev. A 41, 6480 (1990).

[35] See, for example, W. H. Press, S. A. Teukolsky, W. T. Vetterling, and B. P. Flannery, Numerical Recipes in FORTRAN 77 (Cambridge University Press, Cambridge, 1986), Chap. 19.

[36] Ref. [35], Chap. 11.

[37] Ref. [35], p. 140.

[38] S. Chelkowski, M. Zamojski, and A. D. Bandrauk, Phys. Rev. A 63, 023409 (2001).

[39] A. Baltuška, Th. Udem, M. Uiberacker, M. Hentschel, E. Goulielmakis, Ch. Gohle, R. Holzwarth, V. S. Yakovlev, A. Scrinzi, T. W. Hänsch, and F. Krausz, Nature (London) 421, 611 (2003).

[40] S. Chelkowski, A. D. Bandrauk, and A. Apolonski, Phys. Rev. A 70, 013815 (2004).

[41] S. X. Hu and A. F. Starace, Phys. Rev. A 68, 043407 (2003).

[42] A. D. Bandrauk, S. Chelkowski, D. J. Diestler, J. Manz, and K.-J. Yuan, Int. J. Mass. Spectrom. 277, 189 (2008).

[43] A. deBohan, P. Antoine, D. B. Milošević, and B. Piraux, Phys. Rev. Lett. 81, 1837 (1998).

[44] G. G. Paulus, F. Lindner, H. Walther, A. Baltuška, E. Goulielmakis, M. Lezius, and F. Krausz, Phys. Rev. Lett. 91, 253004 (2003).

[45] M. Abramowitz and I. A. Stegun, Handbook of Mathematical Functions (Dover Publications, Inc., New York, 1972), Chap. 22. 